TRANSACTIONS OF THE

AMERICAN MATHEMATICAL SOCIETY

Volume 357 , Number 5 , Pages 1877-1899

S 0002-9947(04)03496-8

Article electronically published on July 22, 2004

\title{
POSET FIBER THEOREMS
}

\author{
ANDERS BJÖRNER, MICHELLE L. WACHS, AND VOLKMAR WELKER
}

\begin{abstract}
Suppose that $f: P \rightarrow Q$ is a poset map whose fibers $f^{-1}\left(Q_{\leq q}\right)$ are sufficiently well connected. Our main result is a formula expressing the homotopy type of $P$ in terms of $Q$ and the fibers. Several fiber theorems from the literature (due to Babson, Baclawski and Quillen) are obtained as consequences or special cases. Homology, Cohen-Macaulay, and equivariant versions are given, and some applications are discussed.
\end{abstract}

\section{INTRODUCTION}

In an influential paper Quillen [17] presented several "fiber theorems" for posets (i.e., partially ordered sets). They have the general form: given a poset map $f: P \rightarrow$ $Q$, certain properties can be transferred from $Q$ to $P$ if only the fibers $f^{-1}\left(Q_{\leq q}\right)$ are sufficiently well-behaved. The best known of these results (often referred to as "the Quillen fiber lemma") says that if the fibers are contractible then $P$ has the same homotopy type as $Q$. Another one says that if $Q$ and all fibers are homotopy CohenMacaulay (and some other conditions are met), then so is $P$. The corresponding fiber theorem for transferring ordinary Cohen-Macaulayness from $Q$ to $P$ was given around the same time by Baclawski 2].

The Quillen fiber lemma has become a fundamental tool in topological combinatorics, frequently used to determine homotopy type or compute homology of combinatorial complexes. In this paper we present a generalization which subsumes several of the known fiber theorems.

We now proceed to state the main result. Then we comment on the contents of the rest of the paper and on some related work. First, however, a few definitions are needed.

All posets in this paper are assumed to be finite. For any element $x$ of a poset $P$ we let $P_{>x}:=\{y \in P \mid y>x\}$ and $P_{\geq x}:=\{y \in P \mid y \geq x\}$. The subsets $P_{<x}$ and $P_{\leq x}$ are defined similarly. Define the length $\ell(P)$ to be the length of a longest chain of $P$, where the length of a chain is one less than its number of elements. In particular, the length of the empty poset is -1 . Given two posets $P$ and $Q$, a

Received by the editors July 25, 2002 and, in revised form, August 20, 2003.

2000 Mathematics Subject Classification. Primary 05E25, 06A11, 55P10.

The first author was supported by Göran Gustafsson Foundation for Research in Natural Sciences and Medicine, and by EC's IHRP programme, grant HPRN-CT-2001-00272.

The second author was supported in part by National Science Foundation grants DMS 9701407 and DMS 0073760.

The third author was supported by Deutsche Forschungsgemeinschaft (DFG), and by EC's IHRP programme, grant HPRN-CT-2001-00272. 
map $f: P \rightarrow Q$ is called a poset map if it is order preserving, i.e., $x \leq_{P} y$ implies $f(x) \leq_{Q} f(y)$.

The order complex $\Delta(P)$ of a poset $P$ is defined to be the abstract simplicial complex whose faces are the chains of $P$. Usually we do not distinguish notationally between an abstract simplicial complex $\Delta$ and its geometric realization $\|\Delta\|$. The distinction should be understood from the context. Clearly, $\operatorname{dim} \Delta(P)=\ell(P)$. The join of simplicial complexes (or topological spaces) is denoted by $*$ and wedges are denoted by $\vee$.

A topological space $X$ is said to be $r$-connected (for $r \geq 0$ ) if it is nonempty and connected and its $j$ th homotopy group $\pi_{j}(X)$ is trivial for all $j=1, \ldots, r$. A nonempty space $X$ is said to be $r$-acyclic if its $j$ th reduced integral homology group $\tilde{H}_{j}(X)$ is trivial for all $j=0,1, \ldots, r$. We say that $X$ is $(-1)$-connected and $(-1)$-acyclic when $X$ is nonempty. It is also convenient (for later use) to define that every space is $r$-connected and $r$-acyclic for all $r \leq-2$.

We use the notation $\simeq$ to denote homotopy equivalence and $\cong$ to denote group or vector space isomorphism. The $j$ th reduced simplicial integral homology of the order complex of a poset $P$ is denoted by $\tilde{H}_{j}(P)$.

The following is the basic version of our main result. More general versions appear in Theorems 2.5 and 2.7 .

Theorem 1.1. Let $f: P \rightarrow Q$ be a poset map such that for all $q \in Q$ the fiber $\Delta\left(f^{-1}\left(Q_{\leq q}\right)\right)$ is $\ell\left(f^{-1}\left(Q_{<q}\right)\right)$-connected. Then

$$
\Delta(P) \simeq \Delta(Q) \vee\left\{\Delta\left(f^{-1}\left(Q_{\leq q}\right)\right) * \Delta\left(Q_{>q}\right) \mid q \in Q\right\},
$$

where $\vee$ denotes the wedge (of $\Delta(Q)$ and all $\Delta\left(f^{-1}\left(Q_{\leq q}\right)\right) * \Delta\left(Q_{>q}\right)$ ) formed by identifying the vertex $q$ in $\Delta(Q)$ with any element of $f^{-1}\left(Q_{\leq q}\right)$, for each $q \in Q$. Consequently, if $\Delta(Q)$ is connected, then

$$
\Delta(P) \simeq \Delta(Q) \vee \bigvee_{q \in Q} \Delta\left(f^{-1}\left(Q_{\leq q}\right)\right) * \Delta\left(Q_{>q}\right) .
$$

We will refer to a poset map $f: P \rightarrow Q$ such that for all $q \in Q$ the fiber $\Delta\left(f^{-1}\left(Q_{\leq q}\right)\right)$ is $\ell\left(f^{-1}\left(Q_{<q}\right)\right)$-connected as being well-connected. Note that the connectivity condition implies that each fiber $f^{-1}\left(Q_{\leq q}\right)$ is nonempty.

For clarity, let us remark that if $\Delta(Q)$ is connected, then the space described on the right-hand side of (1.1), which has $|Q|$ wedge-points, is homotopy equivalent to a one-point wedge, where arbitrarily chosen points of $f^{-1}\left(Q_{\leq q}\right)$, one for each $q \in Q$, are identified with some (arbitrarily chosen) point of $Q$. Thus (1.1) can be restated as (1.2) in this case. For general $Q$ one needs at least as many wedge-points as there are connected components of $Q$. So (1.1) can be restated as

$$
\Delta(P) \simeq \biguplus_{i=1}^{k}\left(\Delta\left(Q^{(i)}\right) \vee \bigvee_{q \in Q^{(i)}} \Delta\left(f^{-1}\left(Q_{\leq q}\right)\right) * \Delta\left(Q_{>q}\right)\right)
$$

where $Q^{(1)}, \ldots, Q^{(k)}$ are the connected components of $Q$ and $\biguplus$ denotes disjoint union.

The definition of the join operation used here also needs clarification. The usual definition of $X * Y$ as a quotient of $X \times Y \times I$ (see, e.g., [10, p. 468]) implies that the join is empty if either of $X$ or $Y$ is empty. However, we use another definition in that case, namely $X * \emptyset=\emptyset * X=X$, which agrees with the standard 


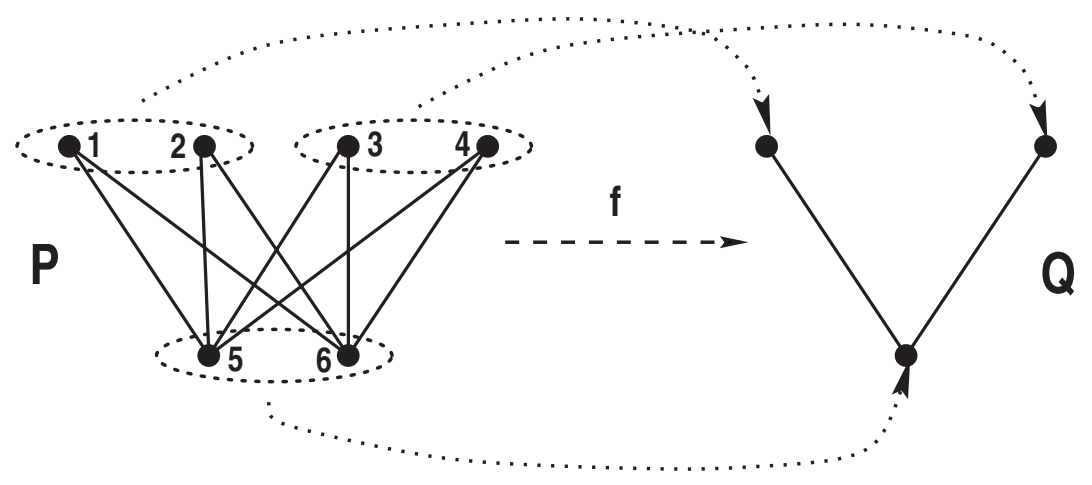

Figure 1. A well-connected poset map

simplicial definition of the join operation. We should also point out that we use the conventions that the empty set is a member of every abstract simplicial complex and that any simplicial map takes the empty set to the empty set. If $P$ is the empty poset, then $\Delta(P)=\{\emptyset\}$.

Example 1.2. Let $f: P \rightarrow Q$ be the poset map depicted in Figure 1. For the two top elements of $Q$ the fiber $\Delta\left(f^{-1}\left(Q_{\leq q}\right)\right)$ is a 1-sphere. For the bottom element of $Q$ the fiber $\Delta\left(f^{-1}\left(Q_{\leq q}\right)\right)$ is a 0 -sphere, and $\Delta\left(Q_{>q}\right)$ is a 0 -sphere too. So in either case $\Delta\left(f^{-1}\left(Q_{\leq q}\right)\right) * \Delta\left(Q_{>q}\right)$ is homeomorphic to a 1 -sphere. Hence the simplicial complex on the right side of (1.1) has a 1-sphere attached to each element of $Q$. Thus Theorem 1.1 determines $\Delta(P)$ to have the homotopy type of a wedge of three 1 -spheres. One can see this directly by observing that $\Delta(P)$ is homeomorphic to two 1-spheres intersecting in two points.

The paper is organized as follows. We prove some generalizations of Theorem 1.1 in Section 2 using the "diagram of spaces" technique. Several corollaries are deduced in Section 3 including generalizations of two results due to Quillen and one due to Babson.

Section 4 gives the homology version of the main result. For an Euler characteristic (Möbius function) version, see Walker [28, Corollary 3.2].

In Section 5 we discuss the non-pure version of the Cohen-Macaulay property, and we prove (based on Theorem 1.1) the generalization to this setting of the Cohen-Macaulay fiber theorems of Baclawski and Quillen.

Section [6] is devoted to an application concerning so-called "inflated" simplicial complexes. In Section 7 we discuss how the Ziegler-Živaljević formula 32 for the homotopy type of the singularity link of an arrangement can be conveniently deduced via Theorem 1.1

The last two sections are devoted to group equivariant versions of Theorem 1.1 In Section 8 we discuss this on the level of equivariant homotopy, and in Section 9 we derive equivariant versions of the homology results.

The need for a fiber result such as Theorem 1.1 arose in the work of the authors. In [26] Wachs uses the results on inflated complexes to compute homotopy type and homology of multigraph matching complexes and wreath product analogues of chessboard complexes (see also [25]). These inflation results have led to other interesting developments such as the work of Pakianathan and Yalçin [15], Shareshian 
[18, and Shareshian and Wachs [19] on complexes related to the Brown complex and the Quillen complex of the symmetric group.

In [9] Björner and Welker use results from this paper to show that certain constructions on posets (the so-called weighted Segre and Rees products, both inspired by ring theoretic constructions in commutative algebra) preserve the CohenMacaulay property, homotopically and over a field. In 27] Wachs uses the equivariant homology version of Theorem 1.1 (Theorem 9.1) to obtain formulas for the representations of wreath product groups acting on the homology of rank selected Dowling lattices.

We are grateful to Vic Reiner, Günter Ziegler and a referee for useful comments on a preliminary version of this paper.

\section{THE PROOF}

In order to prove Theorem 1.1 we need some tools from the theory of diagrams of spaces. This theory was developed in the 60's and 70's by homotopy theorists. Most of the results we need here were originally obtained in this context; however, we take their formulation from [30] since that suits our applications best. We refer the reader to [30] for the original references.

The first combinatorial application of the theory of diagrams of spaces was in the work of Ziegler and Živaljević 32, continued in Welker, Ziegler and Živaljević [30]. Our work is closely related to [32] and [30], and could be said to follow in their footsteps.

A diagram of spaces over a finite poset $Q$ is a functor $\mathcal{D}: Q \rightarrow$ Top from $Q$ into the category of topological spaces. Here we consider $Q$ as a small category with a unique arrow pointing from $x$ to $y$ if $x \leq y$. This means that to each $x \in Q$ we associate a topological space $D_{x}$ and to any pair $x \leq y$ in $Q$ we associate a continuous map $d_{x, y}: D_{x} \rightarrow D_{y}$ such that $d_{x, x}=\operatorname{id}_{D_{x}}$ and $d_{x, z}=d_{y, z} \circ d_{x, y}$ for $x \leq y \leq z$. A simplicial $Q$-diagram is a functor from $Q$ to the category of simplicial complexes. By considering the geometric realization it is clear that a simplicial diagram can be viewed as a diagram of spaces.

There are two constructions of a limit-space associated to a diagram of spaces $\mathcal{D}$.

- colim D: The colimit of the diagram $\mathcal{D}$ is the quotient of the disjoint union $\biguplus_{x \in Q} D_{x}$ modulo the equivalence relation generated by $a \sim b$ if $d_{x, y}(a)=b$ for some $x \leq y$ such that $a \in D_{x}$ and $b \in D_{y}$.

- hocolim $\mathcal{D}$ : The homotopy colimit of the diagram $\mathcal{D}$ is the quotient of the disjoint union $\biguplus_{x \in Q} \Delta\left(Q_{\geq x}\right) \times D_{x}$ modulo the equivalence relation generated by $(c, a) \sim(c, b)$ if $d_{x, y}(a)=b$ for some $x \leq y$ such that $a \in D_{x}$, $b \in D_{y}$ and $c \in \Delta\left(Q_{\geq y}\right)$.

A diagram map $\alpha: \mathcal{D} \rightarrow \mathcal{E}$ is a collection of continuous maps $\alpha_{x}: D_{x} \rightarrow E_{x}$, $x \in Q$, such that $\alpha_{y} \circ d_{x, y}=e_{x, y} \circ \alpha_{x}$ for all $x \leq y$ in $Q$. A diagram map $\alpha: \mathcal{D} \rightarrow \mathcal{E}$ induces a continuous map from hocolim $\mathcal{D}$ to hocolim $\mathcal{E}$ in a natural way.

We need three lemmas from [30]. The first of these is proved in a more general form at the end of this section (Lemma [2.8), and the other two are quoted without proof.

Lemma 2.1 (Homotopy Lemma [30] Lemma 4.6]). Let $\mathcal{D}$ and $\mathcal{E}$ be $Q$-diagrams. Suppose $\alpha: \mathcal{D} \rightarrow \mathcal{E}$ is a diagram map such that $\alpha_{x}: D_{x} \rightarrow E_{x}$ is a homotopy 
equivalence for all $x \in Q$. Then $\alpha$ induces homotopy equivalence,

$$
\text { hocolim } \mathcal{D} \simeq \operatorname{hocolim} \mathcal{E} .
$$

Lemma 2.2 (Wedge Lemma [30 Lemma 4.9], [32, Lemma 1.8]). Let $Q$ be a poset with a minimum element $\hat{0}$ and let $\mathcal{D}$ be a $Q$-diagram. Assume that for each $y>\hat{0}$ in $Q$ there exists a point $c_{y} \in D_{y}$ such that $d_{x, y}(a)=c_{y}$ for all $x<y$ and $a \in D_{x}$. Then

$$
\operatorname{hocolim} \mathcal{D} \simeq \bigvee_{x \in Q}\left(D_{x} * \Delta\left(Q_{>x}\right)\right)
$$

where the wedge is formed by identifying $c_{x} \in D_{x} * \Delta\left(Q_{>x}\right)$ with $x \in D_{\hat{0}} * \Delta\left(Q_{>\hat{0}}\right)$ for all $x>\hat{0}$.

A continuous map $\alpha: X \rightarrow Y$ is said to be a cofibration if for all continuous maps $f_{0}: Y \rightarrow Z$ and homotopies $g_{t}: X \rightarrow Z$ such that $f_{0} \circ \alpha=g_{0}$ there exists a homotopy $f_{t}: Y \rightarrow Z$ such that $g_{t}=f_{t} \circ \alpha$. It is closed if it sends closed sets to closed sets. For example, if $Y$ has a triangulation such that $X$ is triangulated by a subcomplex (one says that $(Y, X)$ is a simplicial pair), then the inclusion map $X \hookrightarrow Y$ is a closed cofibration [10, p. 431].

Lemma 2.3 (Projection Lemma 30, Proposition 3.1]). Let $\mathcal{D}$ be a $Q$-diagram and for each $y \in Q$, let $\mathcal{D}_{<y}$ denote the restriction of $\mathcal{D}$ to $Q_{<y}$. Suppose that the induced map colim $\mathcal{D}_{<y} \rightarrow D_{y}$ is a closed cofibration for all $y$ in $Q$. Then

$$
\text { hocolim } \mathcal{D} \simeq \operatorname{colim} \mathcal{D} \text {. }
$$

The following example of a diagram of spaces is similar to one that appears in Definition 1.2 of [32]. An arrangement of subspaces $\mathcal{A}=\left\{A_{1}, \ldots, A_{m}\right\}$ is a finite collection of closed subspaces of a topological space $U$ such that

(1) $A, B \in \mathcal{A}$ implies that $A \cap B$ is a union of subspaces in $\mathcal{A}$, and

(2) for all $B \in \mathcal{A}$, the inclusion map

$$
\bigcup_{\substack{A \in \mathcal{A} \\ A \subsetneq B}} A \hookrightarrow B
$$

is a cofibration.

Let $Q$ be the inclusion poset $(\mathcal{A}, \subseteq)$. There is an associated $Q$-diagram $\mathcal{D}(\mathcal{A})$, called the subspace diagram of $\mathcal{A}$, which is defined as follows: For each $x \in Q$, let $D_{x}=x$, and for $x \leq y$ let $d_{x, y}$ be the inclusion map $x \hookrightarrow y$. Since the intersection of any pair of subspaces in $\mathcal{A}$ is a union of subspaces in $\mathcal{A}$, it follows that colim $\mathcal{D}(\mathcal{A})$ is homeomorphic to $\bigcup_{A \in \mathcal{A}} A$. On the other hand, by the Projection Lemma hocolim $\mathcal{D}(\mathcal{A}) \simeq \operatorname{colim} \mathcal{D}(\mathcal{A})$. Hence, we get

Corollary 2.4 (to Projection Lemma). Let $\mathcal{A}$ be an arrangement of subspaces. Then

$$
\operatorname{hocolim} \mathcal{D}(\mathcal{A}) \simeq \bigcup_{A \in \mathcal{A}} A
$$

We are now ready to prove Theorem 1.1$]$ which we restate in a slightly more general form. 
Theorem 2.5. Let $f: P \rightarrow Q$ be a poset map such that for all $q \in Q$ the fiber $f^{-1}\left(Q_{\leq q}\right)$ is nonempty, and for all non-minimal $q \in Q$ the inclusion map

$$
\Delta\left(f^{-1}\left(Q_{<q}\right)\right) \hookrightarrow \Delta\left(f^{-1}\left(Q_{\leq q}\right)\right)
$$

is homotopic to a constant map which sends $\Delta\left(f^{-1}\left(Q_{<q}\right)\right)$ to $c_{q}$ for some $c_{q} \in$ $\Delta\left(f^{-1}\left(Q_{\leq q}\right)\right)$. Then

$$
\Delta(P) \simeq \Delta(Q) \vee\left\{\Delta\left(f^{-1}\left(Q_{\leq q}\right)\right) * \Delta\left(Q_{>q}\right) \mid q \in Q\right\},
$$

where the wedge is formed by identifying the vertex $q$ in $\Delta(Q)$ with $c_{q}$, for each $q \in Q$.

Proof. Let $\mathcal{A}=\left\{\Delta\left(f^{-1}\left(Q_{\leq q}\right)\right) \mid q \in Q\right\}$. We claim that $\mathcal{A}$ is an arrangement of subspaces of $\Delta(P)$. For all $x, y \in Q$, we have

$$
\Delta\left(f^{-1}\left(Q_{\leq x}\right)\right) \cap \Delta\left(f^{-1}\left(Q_{\leq y}\right)\right)=\bigcup_{z: z \leq x, y} \Delta\left(f^{-1}\left(Q_{\leq z}\right)\right) .
$$

Let $B \in \mathcal{A}$. Since $\left(B, \bigcup_{\substack{A \in \mathcal{A} \\ A \subset B}} A\right)$ is a simplicial pair, the inclusion map $\bigcup_{\substack{A \in \mathcal{A} \\ A \subset B}} A$ $\hookrightarrow B$ is a cofibration. Hence $\mathcal{A}$ is indeed an arrangement of subspaces. Clearly $\bigcup_{A \in \mathcal{A}} A=\Delta(P)$. Hence by Corollary 2.4

$$
\Delta(P) \simeq \operatorname{hocolim} \mathcal{D}(\mathcal{A}) .
$$

Now let $E_{y}=\Delta\left(f^{-1}\left(Q_{\leq y}\right)\right)$ for all $y \in Q$. For all $x<y$, let $e_{x, y}: E_{x} \rightarrow E_{y}$ be the constant map $e_{x, y}(a)=c_{y}$ for all $a \in E_{x}$. The spaces $E_{y}$ and the maps $e_{x, y}$ form a $Q$-diagram $\mathcal{E}$. Let $\hat{Q}$ be the poset obtained from $Q$ by attaching a minimum element $\hat{0}$ to $Q$, and let $\hat{\mathcal{E}}$ be the $\hat{Q}$-diagram obtained by including $E_{\hat{0}}=\emptyset$ in $\mathcal{E}$. Clearly hocolim $\mathcal{E}=\operatorname{hocolim} \hat{\mathcal{E}}$. It therefore follows from the Wedge Lemma (Lemma 2.2) that

$$
\text { hocolim } \mathcal{E} \simeq \Delta(Q) \vee\left\{\Delta\left(f^{-1}\left(Q_{\leq q}\right)\right) * \Delta\left(Q_{>q}\right) \mid q \in Q\right\},
$$

where the wedge is formed by identifying $c_{q} \in \Delta\left(f^{-1}\left(Q_{\leq q}\right)\right) * \Delta\left(Q_{>q}\right)$ with $q \in$ $\Delta(Q)$, for all $q \in Q$.

It remains to show that

$$
\operatorname{hocolim} \mathcal{D}(\mathcal{A}) \simeq \operatorname{hocolim} \mathcal{E} .
$$

We use the Homotopy Lemma (Lemma 2.11).

Suppose that $y$ is not minimal in $Q$. Consider the homotopy from the inclusion map $\Delta\left(f^{-1}\left(Q_{<y}\right)\right) \hookrightarrow \Delta\left(f^{-1}\left(Q_{\leq y}\right)\right)$ to the constant map which sends $\Delta\left(f^{-1}\left(Q_{<y}\right)\right)$ to $c_{y}$. By the homotopy extension property for simplicial pairs [10, pp. 430-431], such a homotopy can be extended to a homotopy equivalence

$$
\alpha_{y}: \Delta\left(f^{-1}\left(Q_{\leq y}\right)\right) \rightarrow \Delta\left(f^{-1}\left(Q_{\leq y}\right)\right)
$$

which takes $\Delta\left(f^{-1}\left(Q_{<y}\right)\right)$ to $c_{y}$. For minimal $y \in Q$, let $\alpha_{y}$ be the identity mapping on $\Delta\left(f^{-1}\left(Q_{\leq y}\right)\right)$. The Homotopy Lemma applies to the diagram map $\alpha: \mathcal{D}(\mathcal{A}) \rightarrow \mathcal{E}$ and completes the proof.

Proof of Theorem 1.1. The connectivity condition implies that each fiber is nonempty. Since all maps from a triangulable space of dimension $r$ to an $r$-connected space are homotopic, the connectivity condition also implies that the inclusion map $\Delta\left(f^{-1}\left(Q_{<y}\right)\right) \hookrightarrow \Delta\left(f^{-1}\left(Q_{\leq y}\right)\right)$ is homotopic to any constant map. Hence we can apply Theorem 2.5 . 
Remark 2.6. In the corollaries and homology versions of Theorem 1.1 that appear in the following sections, the fiber connectivity condition (or its homology version) can be replaced by the weaker fiber condition (or its homology version) given in Theorem 2.5. For simplicity, we have chosen to use the simpler (albeit stronger) connectivity assumption throughout the paper.

One of Quillen's poset fiber results [17. Proposition 7.6] states that if all the fibers of a poset map $f: P \rightarrow Q$ are $t$-connected, then $\Delta(P)$ is $t$-connected if and only if $\Delta(Q)$ is $t$-connected. A more general result, stating that if all the fibers are $t$-connected then $f$ induces isomorphism of homotopy groups $\pi_{r}(\Delta(P), b) \cong$ $\pi_{r}(\Delta(Q), f(b))$ for all $r \leq t$ and all basepoints $b$, was obtained by Björner [4, p. 1850], 5. Since this does not follow from Theorem 1.1, we ask whether there is a stronger version of Theorem 1.1 which implies these fiber results. We have been able to obtain the following partial answer to this question.

A $t$-equivalence is a continuous map $\psi: X \rightarrow Y$ such that the induced map $\psi_{*}: \pi_{r}(X, b) \rightarrow \pi_{r}(Y, \psi(b))$ is an isomorphism for all $r<t$ and all basepoints $b$, and is a surjection for $r=t$ and all basepoints $b$. By Whitehead's theorem [10] p. 486], the following result implies Theorem 1.1 when $t$ is large.

Theorem 2.7. Let $f: P \rightarrow Q$ be a poset map and let $t$ be a nonnegative integer. If each fiber $\Delta\left(f^{-1}\left(Q_{\leq q}\right)\right)$ is $\min \left\{t, \ell\left(f^{-1}\left(Q_{<q}\right)\right)\right\}$-connected, then there is a t-equivalence

$$
\psi: \Delta(P) \rightarrow \Delta(Q) \vee\left\{\Delta\left(f^{-1}\left(Q_{\leq q}\right)\right) * \Delta\left(Q_{>q}\right) \mid q \in Q\right\} .
$$

Proof. The proof follows the lines of the proof of Theorem 1.1. The diagram map $\alpha: \mathcal{D}(\mathcal{A}) \rightarrow \mathcal{E}$ in the proof of Theorem 2.5 is modified so that $\alpha_{y}$ is the constant map to $c_{y}$ when $t<\ell\left(f^{-1}\left(Q_{<y}\right)\right)$. Using the fact that $\alpha_{y}$ is a $t$-equivalence for all $y$, we complete the proof by applying the following strong version of the homotopy lemma.

Lemma 2.8 (Strong Homotopy Lemma). Let $\mathcal{D}$ and $\mathcal{E}$ be $Q$-diagrams. Suppose $\alpha: \mathcal{D} \rightarrow \mathcal{E}$ is a diagram map such that for each $y \in Q$ the map $\alpha_{y}: D_{y} \rightarrow E_{y}$ is a t-equivalence, where $t$ is some fixed nonnegative integer. Then the induced map from hocolim $\mathcal{D}$ to hocolim $\mathcal{E}$ is a t-equivalence.

Proof. The proof of the homotopy lemma given in the appendix of [32] is modified by using [31, Corollary 2] instead of [32, Corollary 4.2].

We use induction on the size of $Q$. If $|Q|=1$ the result is trivial. Let $|Q|>1$.

Case 1: $Q$ has a unique maximum $y$. The natural collapsing maps hocolim $\mathcal{D} \rightarrow$ $D_{y}$ and hocolim $\mathcal{E} \rightarrow E_{y}$ are deformation retractions which commute with the appropriate maps. So the result holds.

Case 2: $Q$ has more than one maximal element. Let $y$ be one of the maximal elements. Let $\mathcal{D}(<y), \mathcal{D}(\leq y)$ and $\mathcal{D}(\neq y)$ be the restrictions of $\mathcal{D}$ to the posets $Q_{<y}, Q_{\leq y}$ and $Q \backslash\{y\}$, respectively. Let

$$
X=\operatorname{hocolim} \mathcal{D} \quad \text { and } \quad X_{0}=\text { hocolim } \mathcal{D}(\leq y) .
$$

We view $X_{0}$ as the mapping cylinder of the natural map hocolim $\mathcal{D}(<y) \rightarrow D_{y}$. So $X_{0}$ is hocolim $\mathcal{D}(<y) \times[0,1]$ glued to $D_{y}$ as a mapping cylinder. Now view hocolim $\mathcal{D}(\neq y)$ as a space that contains hocolim $D(<y) \times\{0\}$ and set

$$
X_{1}=\operatorname{hocolim} \mathcal{D}(\neq y) \cup(\text { hocolim } \mathcal{D}(<y) \times[0,1 / 2]) .
$$


We have

$$
X_{2}:=X_{0} \cap X_{1}=\text { hocolim } \mathcal{D}(<y) \times[0,1 / 2] .
$$

Clearly hocolim $\mathcal{D}(\neq y)$ is a deformation retract of $X_{1}$, hocolim $\mathcal{D}(<y)$ is a deformation retract of $X_{2}$, and

$$
\stackrel{\circ}{X}_{0} \cup \stackrel{\circ}{X}_{1}=\operatorname{hocolim} \mathcal{D}(\leq y) \cup \operatorname{hocolim} \mathcal{D}(\neq y)=\operatorname{hocolim} \mathcal{D}=X,
$$

where $\stackrel{\circ}{X}_{i}$ denotes the interior of $X_{i}$ in $X$. Define $Y$ and $Y_{i}, i=0,1,2$, analogously for $\mathcal{E}$. Since by induction we have that the maps $X_{i} \rightarrow Y_{i}$ induced by $\alpha$ are $t$ equivalences, we can apply [31, Corollary 2] to conclude that the induced map $X \rightarrow Y$ is also a $t$-equivalence.

\section{Corollaries}

The following is a direct consequence of Theorem 1.1. It is a minor generalization of "the Quillen fiber lemma" [17, Proposition 1.6].

Corollary 3.1. Let $f: P \rightarrow Q$ be a poset map, and suppose that for all $q \in Q$ either the fiber $\Delta\left(f^{-1}\left(Q_{\leq q}\right)\right)$ is contractible or else it is $\ell\left(f^{-1}\left(Q_{<q}\right)\right)$-connected and $\Delta\left(Q_{>q}\right)$ is contractible. Then

$$
\Delta(P) \simeq \Delta(Q) .
$$

Another result of Quillen's [17, Theorem 9.1] can be generalized as follows.

Corollary 3.2. Let $f: P \rightarrow Q$ be a poset map. Fix $t \geq 0$. Suppose for all $q \in Q$ that the fiber $\Delta\left(f^{-1}\left(Q_{\leq q}\right)\right)$ is $\ell\left(f^{-1}\left(Q_{<q}\right)\right)$-connected and that $\Delta\left(Q_{>q}\right)$ is $\left(t-\ell\left(f^{-1}\left(Q_{<q}\right)\right)-2\right)$-connected. Then

$$
\pi_{r}(\Delta(P), b) \cong \pi_{r}(\Delta(Q), f(b))
$$

for all $r \leq t$ and all basepoints $b$. Consequently, $\Delta(P)$ is $t$-connected if and only if $\Delta(Q)$ is t-connected.

Proof. Using the fact that the join of an $i$-connected simplicial complex with a $j$ connected simplicial complex is $(i+j+2)$-connected, we find that all components of the wedge on the right-hand side of equation (1.1) are $t$-connected with the possible exception of $\Delta(Q)$. We claim that the $t$-connectivity of these components implies that for all $r \leq t$ and all $b \in P$,

$$
\pi_{r}(\Delta(Q), f(b)) \cong \pi_{r}(\Gamma, f(b)),
$$

where $\Gamma$ is the simplicial complex on the right-hand side of (1.1). To establish this claim we use the following homotopy theory fact, which can be proved by using 13 . Theorem 6.2], [11, Exercise 4.1.15] and Van Kampen's theorem: If $X$ is a connected $\mathrm{CW}$-complex and $Y$ is a $t$-connected CW-complex, then $\pi_{r}(X \vee Y) \cong \pi_{r}(X)$ for all $r \leq t$.

Remark 3.3. After this paper was finished G. Ziegler pointed us to the recent paper 14], which contains two results (Theorem 3.8 and Theorem 3.6) very similar to our Corollary 3.2 and its homology version.

In his thesis, Babson [1] (see also [21, Lemma 3.2]) presented a fiber lemma for posets involving fibers of the form $f^{-1}(q)$. It can be generalized as follows. (Babson's lemma is the special case where condition (i) is sharpened to " $\Delta\left(f^{-1}(q)\right)$ is contractible", condition (ii) to " $\Delta\left(f^{-1}(q) \cap P_{\geq p}\right)$ is contractible for all $p \in$ $f^{-1}\left(Q_{\leq q}\right)$ " and the conclusion to " $\Delta(P) \simeq \Delta(Q)$ ".) 
Corollary 3.4. Let $f: P \rightarrow Q$ be a poset map. Suppose that, for every $q \in Q$,

(i) $\Delta\left(f^{-1}(q)\right)$ is $\ell\left(f^{-1}\left(Q_{<q}\right)\right)$-connected, and

(ii) $\Delta\left(f^{-1}(q) \cap P_{\geq p}\right)$ is contractible or else it is $\ell\left(f^{-1}(q) \cap P_{>p}\right)$-connected and $\Delta\left(P_{<p}\right)$ is contractible, for all $p \in f^{-1}\left(Q_{\leq q}\right)$.

Then

$$
\Delta(P) \simeq \Delta(Q) \vee\left\{\Delta\left(f^{-1}(q)\right) * \Delta\left(Q_{>q}\right) \mid q \in Q\right\},
$$

where the wedge is formed by identifying the vertex $q \in \Delta(Q)$ with any element of $f^{-1}(q)$, for each $q \in Q$.

Proof. By Theorem 1.1 and condition (i) it suffices to show that the poset inclusion map

$$
g: f^{-1}(q) \rightarrow f^{-1}\left(Q_{\leq q}\right)
$$

induces homotopy equivalence of order complexes. But this follows from Corollary 3.1 and condition (ii), since

$$
\begin{gathered}
g^{-1}\left(\left(f^{-1}\left(Q_{\leq q}\right)\right)_{\geq p}\right)=f^{-1}(q) \cap P_{\geq p}, \\
g^{-1}\left(\left(f^{-1}\left(Q_{\leq q}\right)\right)_{>p}\right)=f^{-1}(q) \cap P_{>p}
\end{gathered}
$$

and $f^{-1}\left(Q_{\leq q}\right)_{<p}=P_{<p}$.

A simplicial complex version of Theorem 1.1 follows from the poset version. Given a face $F$ of a simplicial complex $\Delta$, let $\dot{F}$ denote the subcomplex of faces contained in $F$ and let $\operatorname{lk}_{\Delta} F$ denote the link of $F$, i.e.,

$$
\mathrm{lk}_{\Delta} F=\{G \in \Delta \mid G \cap F=\emptyset \text { and } G \cup F \in \Delta\} .
$$

Corollary 3.5. Let $f: \Gamma \rightarrow \Delta$ be a simplicial map. If the fiber $f^{-1}(\dot{F})$ is $\operatorname{dim} f^{-1}(\dot{F} \backslash\{F\})$-connected for all nonempty faces $F$ of $\Delta$, then

$$
\Gamma \simeq \Delta \vee\left\{f^{-1}(\dot{F}) * \mathrm{lk}_{\Delta} F \mid F \in \Delta \backslash\{\emptyset\}\right\},
$$

where the wedge is formed by identifying a vertex of $f^{-1}(\dot{F})$ with a vertex of $F$ for each nonempty face $F$ of $\Delta$.

Proof. We view $f$ as a poset map from the poset of nonempty faces of $\Gamma$ to the poset of nonempty faces of $\Delta$. Since the barycentric subdivision of a complex is homeomorphic to the complex, $f$ is a well-connected poset map. Hence by Theorem 1.1

$$
\operatorname{sd} \Gamma \simeq \operatorname{sd} \Delta \vee\left\{\operatorname{sd} f^{-1}(\dot{F}) * \operatorname{sdlk}_{\Delta} F \mid F \in \Delta \backslash\{\emptyset\}\right\},
$$

where sd denotes the barycentric subdivision. Passing from the barycentric subdivision to the original complexes yields the result.

\section{Homology fiBer theOREM}

This section is devoted to the homology version of Theorem 1.1 and its corollaries. For the proofs we again rely on the theory of diagrams of spaces. Homology versions of the tools of Section 2 were used by Sundaram and Welker [22], and we refer to their paper for further details.

We use the notation $\tilde{H}_{[t]}(\Delta)=\bigoplus_{i \leq t} \tilde{H}_{i}(\Delta)$. 
Theorem 4.1. Fix an integer $t \geq 0$. Let $f: P \rightarrow Q$ be a poset map such that for all $q \in Q$ the fiber $\Delta\left(f^{-1}\left(Q_{\leq q}\right)\right)$ is $\min \left\{t, \ell\left(f^{-1}\left(Q_{<q}\right)\right)\right\}$-acyclic and either $\tilde{H}_{[t]}\left(f^{-1}\left(Q_{\leq q}\right)\right)$ or $\tilde{H}_{[t]}\left(Q_{>q}\right)$ is free. Then for all $r \leq t$,

$$
\tilde{H}_{r}(P) \cong \tilde{H}_{r}(Q) \oplus \bigoplus_{q \in Q} \bigoplus_{i=-1}^{r}\left(\tilde{H}_{i}\left(f^{-1}\left(Q_{\leq q}\right)\right) \otimes \tilde{H}_{r-i-1}\left(Q_{>q}\right)\right) .
$$

The same result holds for homology taken over any field.

For the proof we use a slight generalization of a homology version of the Wedge Lemma due to Sundaram and Welker [22. It will be proved later in this paper as a special case of Proposition 9.8, see also Remark 9.9

Proposition 4.2 (22, Proposition 2.3]). Let $\mathcal{D}$ be a simplicial $Q$-diagram for which each $D_{x} \neq\{\emptyset\}$. Let $t$ be a nonnegative integer. Assume that for all nonminimal $y$ in $Q$ and $r \leq t$, the induced map

$$
\left(\bigcup_{x<y} d_{x, y}\right)^{*}: \tilde{H}_{r}\left(\biguplus_{x<y} D_{x}\right) \rightarrow \tilde{H}_{r}\left(D_{y}\right)
$$

is trivial. Assume also that either $\tilde{H}_{[t]}\left(D_{x}\right)$ or $\tilde{H}_{[t]}\left(Q_{>x}\right)$ is free for all $x$. Then for all $r \leq t$,

$$
\tilde{H}_{r}(\operatorname{hocolim} \mathcal{D}) \cong \tilde{H}_{r}(Q) \oplus \bigoplus_{x \in Q} \bigoplus_{i=-1}^{r}\left(\tilde{H}_{i}\left(D_{x}\right) \otimes \tilde{H}_{r-i-1}\left(Q_{>x}\right)\right) .
$$

The same result holds for homology taken over any field.

Proof of Theorem 4.1. Let $\mathcal{D}(\mathcal{A})$ be the simplicial $Q$-diagram described in the proof of Theorem 2.5. The map

$$
\left(\bigcup_{x<y} d_{x, y}\right)^{*}: \tilde{H}_{r}\left(\biguplus_{x<y} f^{-1}\left(Q_{\leq x}\right)\right) \rightarrow \tilde{H}_{r}\left(f^{-1}\left(Q_{\leq y}\right)\right)
$$

induced by the inclusion map is trivial for all $r \leq t$, since $\tilde{H}_{r}\left(\biguplus_{x<y} f^{-1}\left(Q_{\leq x}\right)\right)=0$ if $r>\ell\left(f^{-1}\left(Q_{<y}\right)\right)$ for dimensional reasons, and $\tilde{H}_{r}\left(f^{-1}\left(Q_{\leq y}\right)\right)=0$ for all $r \leq$ $\min \left\{t, \ell\left(f^{-1}\left(Q_{<y}\right)\right)\right\}$ by the acyclicity assumption. Thus we can apply Proposition 4.2 to $\mathcal{D}(\mathcal{A})$. The result now follows from equation (2.1).

Homology versions of Corollaries 3.1-3.5 follow straightforwardly. We state two of them.

Corollary 4.3. Let $f: P \rightarrow Q$ be a poset map. Suppose that for all $q \in Q$ either the fiber $\Delta\left(f^{-1}\left(Q_{\leq q}\right)\right)$ is $t$-acyclic or else it is $\ell\left(f^{-1}\left(Q_{<q}\right)\right)$-acyclic and $\Delta\left(Q_{>q}\right)$ is t-acyclic. Then

$$
\tilde{H}_{r}(P) \cong \tilde{H}_{r}(Q)
$$

for all $r \leq t$. The same result holds for homology taken over any field.

Corollary 4.4. Let $f: \Gamma \rightarrow \Delta$ be a simplicial map. Suppose that the fiber $f^{-1}(\dot{F})$ is $\min \left\{t, \operatorname{dim} f^{-1}(\dot{F} \backslash\{F\})\right\}$-acyclic and either $\tilde{H}_{[t]}\left(f^{-1}(\dot{F})\right)$ or $\tilde{H}_{[t]}\left(\mathrm{lk}_{\Delta} F\right)$ is free for all nonempty faces $F$ of $\Delta$. Then for all $r \leq t$,

$$
\tilde{H}_{r}(\Gamma) \cong \tilde{H}_{r}(\Delta) \oplus \bigoplus_{F \in \Delta \backslash\{\emptyset\}} \bigoplus_{i=-1}^{r}\left(\tilde{H}_{i}\left(f^{-1}(\dot{F})\right) \otimes \tilde{H}_{r-i-1}\left(\mathrm{lk}_{\Delta} F\right)\right) .
$$

The same result holds for homology taken over any field. 


\section{Cohen-Macaulay FiBer theorem}

Since the late 1970's two very similar fiber theorems for transferring the CohenMacaulay property of posets are known, one for the homology version and one for the homotopy version, due to Baclawski [2] and Quillen [17], respectively. Several years later Stanley [20] introduced the more general property of "sequential Cohen-Macaulayness". In this section we introduce a homotopy version of the sequential Cohen-Macaulay property by considering a characterization of sequential Cohen-Macaulayness due to Wachs 24. We show how the homology and homotopy versions of the sequential Cohen-Macaulay property can be transferred via certain poset maps, thereby reproving and generalizing the results of Baclawski and Quillen.

Let $\Delta$ be a simplicial complex, and for $0 \leq m \leq \operatorname{dim} \Delta$ let $\Delta^{\langle m\rangle}$ be the subcomplex generated by all facets (i.e. maximal faces) of dimension at least $m$. We say that $\Delta$ is sequentially connected if $\Delta^{\langle m\rangle}$ is $(m-1)$-connected for all $m=0,1, \ldots, \operatorname{dim} \Delta$. Similarly, we say that $\Delta$ is sequentially acyclic over $\mathbf{k}$ if $\tilde{H}_{r}(\Delta\langle m\rangle ; \mathbf{k})=0$ for all $r<m \leq \operatorname{dim} \Delta$, where $\mathbf{k}$ is the ring of integers or a field.

A simplicial complex is said to be pure if all facets are of equal dimension. Clearly a pure $d$-dimensional simplicial complex is sequentially connected if and only if it is $(d-1)$-connected, and it is sequentially acyclic if and only if it is $(d-1)$-acyclic. Cohen-Macaulay (CM) complexes (see 20]) are pure. The notion of sequentially Cohen-Macaulay (SCM) simplicial complexes is a nonpure generalization due to Stanley [20, Chap. III, Sec. 2]. In Wachs [24, Theorem 1.5] the following characterization is given: a simplicial complex is SCM over $\mathbf{k}$ if and only if the link of each of its faces is sequentially acyclic over $\mathbf{k}$. (The term "vanishing homology property" was used in place of "sequentially acyclic" in 24].) A simplicial complex is CM if and only if it is SCM and pure.

One can formulate a homotopy version of the SCM property as follows. We say that $\Delta$ is sequentially homotopy Cohen-Macaulay (SHCM) if the link of each of its faces is sequentially connected. For pure simplicial complexes, SHCM reduces to the notion of homotopy Cohen-Macaulay (HCM). The following sequence of implications holds:

(nonpure) shellable $\Longrightarrow \mathrm{SHCM} \Longrightarrow \mathrm{SCM}$ over $\mathbb{Z} \Longrightarrow \mathrm{SCM}$ over $\mathbf{k}$, for all fields $\mathbf{k}$.

For more information about $\mathrm{S}(\mathrm{H}) \mathrm{CM}$ complexes, see [8].

A poset is said to be $\mathrm{CM}$ (SCM, HCM or SHCM) if its order complex is. A poset $P$ is said to be semipure if all closed principal lower order ideals $P_{\leq x}$ are pure. The rank, $\operatorname{rk}(x)$, of an element $x$ in a semipure poset $P$ is defined to be $\ell\left(P_{\leq x}\right)$. Finally, $P^{\langle m\rangle}$ denotes the lower order ideal of $P$ generated by elements of rank at least $m$.

In the pure case part (i) of the following result specializes to the homotopy CohenMacaulay fiber theorem of Quillen [17, Corollary 9.7], and part (ii) specializes to Baclawski's Cohen-Macaulay fiber theorem [2, Theorem 5.2].

Theorem 5.1. Let $P$ and $Q$ be semipure posets and let $f: P \rightarrow Q$ be a surjective rank-preserving poset map.

(i) Assume that for all $q \in Q$ the fiber $\Delta\left(f^{-1}\left(Q_{\leq q}\right)\right)$ is HCM. If $Q$ is SHCM, then so is $P$.

(ii) Let $\mathbf{k}$ be a field or $\mathbb{Z}$, and assume that for all $q \in Q$ the fiber $\Delta\left(f^{-1}\left(Q_{\leq q}\right)\right)$ is $C M$ over $\mathbf{k}$. If $Q$ is $S C M$ over $\mathbf{k}$, then so is $P$. 
(iii) If the conditions of (i) or (ii) are fulfilled, then

$$
\begin{gathered}
\beta_{i}(P)=\beta_{i}(Q)+\sum_{q \in Q^{\langle i\rangle}} \beta_{\operatorname{rk}(q)}\left(f^{-1}\left(Q_{\leq q}\right)\right) \beta_{i-\operatorname{rk}(q)-1}\left(Q_{>q}\right) . \\
\text { [Here } \beta_{i}(\cdot)=\operatorname{rank} \tilde{H}_{i}(\cdot) \text {, or } \beta_{i}(\cdot)=\operatorname{dim}_{\mathbf{k}} \tilde{H}_{i}(\cdot ; \mathbf{k}) \text { if } \mathbf{k} \text { is a field.] }
\end{gathered}
$$

Proof. We begin with part (i). First we show that $P$ is sequentially connected; that is, $\Delta(P)^{\langle m\rangle}$ is $(m-1)$-connected for all $m=0, \ldots, \ell(P)$. Since $P \neq \emptyset$, we may assume that $m>0$. Note that $\Delta(P)^{\langle m\rangle}=\Delta\left(P^{\langle m\rangle}\right)$. We will show that Corollary 3.2 (with $t=m-1$ ) applies to the map $f^{\langle m\rangle}: P^{\langle m\rangle} \rightarrow Q^{\langle m\rangle}$, where $f^{\langle m\rangle}$ is the restriction of $f$.

We claim that for all $q \in Q^{\langle m\rangle}$,

$$
\left(f^{\langle m\rangle}\right)^{-1}\left(Q_{\leq q}^{\langle m\rangle}\right)=f^{-1}\left(Q_{\leq q}\right),
$$

where $Q_{\leq q}^{\langle m\rangle}:=\left(Q^{\langle m\rangle}\right)_{\leq q}$. To see this, first observe that $Q_{\leq q}^{\langle m\rangle}=Q_{\leq q}$ and

$$
\left(f^{\langle m\rangle}\right)^{-1}\left(Q_{\leq q}^{\langle m\rangle}\right)=f^{-1}\left(Q_{\leq q}\right) \cap P^{\langle m\rangle} .
$$

Hence to establish (5.1) it suffices to show that $f^{-1}\left(Q_{\leq q}\right) \subseteq P^{\langle m\rangle}$. Let $x \in$ $f^{-1}\left(Q_{\leq q}\right)$. Since $q \in Q^{\langle m\rangle}$, there is some $z \in Q$ such that $\operatorname{rk}(z) \geq m$ and $q \leq z$. It follows from the fact that $f$ is surjective and rank-preserving that $f^{-1}\left(Q_{\leq z}\right)$ has a maximal element of $\operatorname{rank} \operatorname{rk}(z)$. Since $f^{-1}\left(Q_{\leq z}\right)$ is pure, all maximal elements have $\operatorname{rank} \operatorname{rk}(z)$. It follows that $x$ is less than or equal to some element of $\operatorname{rank} \operatorname{rk}(z)$. Hence $x \in P^{\langle m\rangle}$, and (5.1) holds. A similar argument yields

$$
\left(f^{\langle m\rangle}\right)^{-1}\left(Q_{<q}^{\langle m\rangle}\right)=f^{-1}\left(Q_{<q}\right)
$$

for all $q \in Q^{\langle m\rangle}$.

Since $f$ is rank-preserving and surjective, we have

$$
\ell\left(\left(f^{\langle m\rangle}\right)^{-1}\left(Q_{<q}^{\langle m\rangle}\right)\right)=\ell\left(f^{-1}\left(Q_{<q}\right)\right)=\ell\left(f^{-1}\left(Q_{\leq q}\right)\right)-1=\operatorname{rk}(q)-1
$$

for all $q \in Q^{\langle m\rangle}$. It follows that $\Delta\left(f^{-1}\left(Q_{\leq q}\right)\right)$ is $\ell\left(f^{-1}\left(Q_{<q}\right)\right)$-connected, since it is HCM. Hence by (5.1) and (5.2), $\Delta\left(\left(f^{\langle m\rangle}\right)^{-1}\left(Q_{\leq q}^{\langle m\rangle}\right)\right)$ is $\ell\left(\left(f^{\langle m\rangle}\right)^{-1}\left(Q_{<q}^{\langle m\rangle}\right)\right)$-connected for all $q \in Q^{\langle m\rangle}$.

On the other hand, note that $\left(Q^{\langle m\rangle}\right)_{>q}=\left(Q_{>q}\right)^{\langle m-\mathrm{rk}(q)-1\rangle}$ for all $q \in Q^{\langle m\rangle}$. Since $\Delta\left(Q_{>q}\right)$ is the link of a face of $\Delta(Q)$, we have that $\Delta\left(Q_{>q}\right)$ is sequentially connected. Hence $\Delta\left(\left(Q_{>q}\right)^{\langle m-\mathrm{rk}(q)-1\rangle}\right)$ is $(m-\operatorname{rk}(q)-2)$-connected. Therefore by (5.3), $\Delta\left(\left(Q^{\langle m\rangle}\right)_{>q}\right)$ is $\left(m-\ell\left(\left(f^{\langle m\rangle}\right)^{-1}\left(Q_{<q}^{\langle m\rangle}\right)\right)-3\right)$-connected.

We have shown that Corollary 3.2 applies. Therefore $\Delta(P)^{\langle m\rangle}=\Delta\left(P^{\langle m\rangle}\right)$ is $(m-1)$-connected, since $\Delta(Q)^{\langle m\rangle}=\Delta\left(Q^{\langle m\rangle}\right)$ is.

Next we check that all open intervals and principal upper and lower order ideals of $P$ are sequentially connected. From this it will follow that the link of every face of $\Delta(P)$ is sequentially connected, since the join of sequentially connected complexes is sequentially connected. (This fact is easy to verify when at most one of the complexes is nonpure, which is the situation here. It is proved in general in [8.)

Let $(a, b)$ be an open interval in $P$. Then $(a, b)$ is an open interval in the fiber $f^{-1}\left(Q_{\leq f(b)}\right)$. Since the fiber is HCM, it follows that $(a, b)$, which is the link of a face of the fiber, is sequentially connected. The same argument works for open principal lower order ideals in $P$. 
To show that all open principal upper order ideals $P_{>x}$ are sequentially connected, we show that the restriction of $f$ to $P_{>x}$ is a surjective rank-preserving poset map onto $Q_{>f(x)}$ whose fibers are HCM. It will then follow by induction that $P_{>x}$ is SHCM (and hence sequentially connected), since $Q_{>f(x)}$ is. The restriction is clearly rank-preserving. The fibers have the form $f^{-1}\left(Q_{\leq q}\right) \cap P_{>x}$, where $q>f(x)$. Since $f^{-1}\left(Q_{\leq q}\right) \cap P_{>x}$ is an open principal upper order ideal of the HCM poset $f^{-1}\left(Q_{\leq q}\right)$, it is HCM. Since $f$ is rank-preserving and surjective and $f^{-1}\left(Q_{\leq q}\right)$ is pure, we have that all the maximal elements of $f^{-1}\left(Q_{\leq q}\right)$ map to $q$. One of these maximal elements must be greater than $x$. Hence there is an element in $P_{>x}$ that maps to $q$. It follows that the restriction of $f$ to $P_{>x}$ is surjective onto $Q_{>f(x)}$.

Part (ii) is proved the same way, using Theorem 4.1 instead of Corollary 3.2 The statement about $\beta_{i}(P)$ in part (iii) is implied by Theorem 4.1 and the fact that $\ell\left(Q_{>q}\right)<i-\operatorname{rk}(q)-1$ for $q \notin Q^{\langle i\rangle}$.

We have the following partial converse to (i) and (ii) of Theorem 5.1. Its proof is similar to that of Theorem 5.1 and is left as an exercise.

Theorem 5.2. Let $P$ and $Q$ be semipure posets and let $f: P \rightarrow Q$ be a surjective rank-preserving poset map. Assume for all $q \in Q$ that the fiber $f^{-1}\left(Q_{<q}\right)$ is $H C M$ (alt. $C M$ ) and that $f^{-1}\left(Q_{>q}\right)=P_{>p}$ for some $p \in P$. If $P$ is $S H C M$ (alt. $\left.S C M\right)$, then so is $Q$.

Corollary 5.3. Let $f: \Gamma \rightarrow \Delta$ be a surjective dimension-preserving simplicial map such that for all faces $F$ of $\Delta$ the fiber $f^{-1}(\dot{F})$ is $H C M$ (alt. CM). If $\Delta$ is SHCM (alt. SCM), then so is $\Gamma$. Conversely, suppose also that for each face $F$ of $\Delta$ the complex $f^{-1}\left(\mathrm{lk}_{\Delta} F\right)$ is the link of some face of $\Gamma$. If $\Gamma$ is $S H C M$ (alt. SCM), then so is $\Delta$.

Proof. This follows from the fact that a simplicial complex is SHCM (alt. SCM) if and only if its barycentric subdivision is.

\section{INFLATED SIMPLICIAL COMPLEXES}

Let $\Delta$ be a simplicial complex on the vertex set $[n]:=\{1,2, \ldots, n\}$, and let $\mathbf{m}=\left(m_{1}, \ldots, m_{n}\right)$ be a sequence of positive integers. We form a new simplicial complex $\Delta_{\mathbf{m}}$, called the $\mathbf{m}$-inflation of $\Delta$, as follows. The vertex set of $\Delta_{\mathbf{m}}$ is $\left\{(i, c) \mid i \in[n], c \in\left[m_{i}\right]\right\}$ and the faces of $\Delta_{\mathbf{m}}$ are of the form $\left\{\left(i_{1}, c_{1}\right), \ldots,\left(i_{k}, c_{k}\right)\right\}$, where $\left\{i_{1}, \ldots, i_{k}\right\}$ is a $k$-element face of $\Delta$ and $c_{j} \in\left[m_{i_{j}}\right]$ for all $j=1, \ldots, k$. We can think of $c_{j}$ as a color assigned to vertex $i_{j}$ and of $\left\{\left(i_{1}, c_{1}\right), \ldots,\left(i_{k}, c_{k}\right)\right\}$ as a coloring of the vertices of face $\left\{i_{1}, \ldots, i_{k}\right\}$. A color for vertex $i$ is chosen from $m_{i}$ colors.

Example 6.1. Let $P$ and $Q$ be the posets depicted in Figure 1 of Section 1 We have that $\Delta(P)$ is the $(2,2,2)$-inflation of $\Delta(Q)$.

Inflated simplicial complexes arose in work of Wachs [26] on bounded degree digraph and multigraph complexes, where the following consequence of Theorem 1.1 is used. This result, for the special case that $\mathbf{m}=(2, \ldots, 2)$, first appeared in Björner [3] pp. 354-355] in connection with subspace arrangements. 
Theorem 6.2. Let $\Delta$ be a simplicial complex on vertex set [n], and let $\mathbf{m}$ be a sequence of $n$ positive integers. If $\Delta$ is connected, then

$$
\Delta_{\mathbf{m}} \simeq \bigvee_{F \in \Delta}\left(\operatorname{susp}^{|F|}\left(\mathrm{lk}_{\Delta} F\right)\right)^{\vee \nu(F, \mathbf{m})},
$$

where $\nu(F, \mathbf{m})=\prod_{i \in F}\left(m_{i}-1\right)$. For general $\Delta$,

$$
\Delta_{\mathbf{m}} \simeq \biguplus_{i=1}^{k} \bigvee_{F \in \Delta^{(i)}}\left(\operatorname{susp}^{|F|}\left(\mathrm{lk}_{\Delta^{(i)}} F\right)\right)^{\vee \nu(F, \mathbf{m})},
$$

where $\Delta^{(1)}, \ldots, \Delta^{(k)}$ are the connected components of $\Delta$.

Proof. Let $f: \Delta_{\mathbf{m}} \rightarrow \Delta$ be the simplicial map that sends each vertex $(i, c)$ of $\Delta_{\mathbf{m}}$ to vertex $i$ of $\Delta$. We call this map the deflating map and show that it is well-connected. We claim that each fiber $f^{-1}(\dot{F})$ is a wedge of $\nu(F, \mathbf{m})$ spheres of $\operatorname{dimension} \operatorname{dim} F$. First observe that the fiber $f^{-1}(\dot{F})$ is a matroid complex. Since all matroid complexes are (pure) shellable [16], the fiber is a wedge of spheres of dimension $\operatorname{dim} F$ (cf. [7]). To determine the number of spheres in the wedge we compute the reduced Euler characteristic. The number of $(k-1)$-dimensional faces in $f^{-1}(\dot{F})$ is $\sum_{A \in\left(\begin{array}{c}F \\ k\end{array}\right)} \prod_{i \in A} m_{i}$. Hence the reduced Euler characteristic of $f^{-1}(\dot{F})$ is

$$
\tilde{\chi}\left(f^{-1}(\dot{F})\right)=\sum_{A \subseteq F}(-1)^{|A|-1} \prod_{i \in A} m_{i}=\prod_{i \in F}\left(1-m_{i}\right) .
$$

Therefore the number of spheres in the wedge is $\left|\tilde{\chi}\left(f^{-1}(\dot{F})\right)\right|=\nu(F, \mathbf{m})$.

We may assume $\Delta$ is connected since the general case follows from this case. By Corollary 3.5,

$$
\Delta_{\mathbf{m}} \simeq \bigvee_{F \in \Delta}\left(S^{\operatorname{dim} F}\right)^{\vee \nu(F, \mathbf{m})} * \mathrm{lk}_{\Delta} F .
$$

The result now follows from the fact that the join operation is distributive over the wedge operation.

Let $\mathbf{k}$ be a field or the ring of integers.

Corollary 6.3. For all $r \in \mathbb{Z}$,

$$
\tilde{H}_{r}\left(\Delta_{\mathbf{m}} ; \mathbf{k}\right)=\bigoplus_{F \in \Delta} \nu(F, \mathbf{m}) \tilde{H}_{r-|F|}\left(\mathrm{lk}_{\Delta} F ; \mathbf{k}\right) .
$$

Corollary 6.4. For any simplicial complex $\Delta$ on $[n]$ and $n$-sequence of positive integers $\mathbf{m}$, the inflated simplicial complex $\Delta_{\mathbf{m}}$ is CM over $\mathbf{k}$ (SCM over $\mathbf{k}, H C M$ or SHCM) if and only if $\Delta$ is.

Proof. This follows from the fact that all the fibers of the deflating map given in the proof of Theorem 6.2 are homotopy Cohen-Macaulay and Corollary 5.3.

Remark 6.5. A poset $P$ is said to be obtained by replicating elements of a poset $Q$ if there is a surjective poset map $f: P \rightarrow Q$ such that (1) $f\left(x_{1}\right)<f\left(x_{2}\right)$ if and only if $x_{1}<x_{2}$ and $(2) f^{-1}(y)$ is an antichain for all $y \in Q$. For example, the poset $P$ of Figure 1 is obtained by replicating elements of $Q$. This operation was shown by Baclawski [2, Theorem 7.3] to preserve CMness. It is easy to see that for any posets $P$ and $Q$, the order complex $\Delta(P)$ is an inflation of the order complex $\Delta(Q)$ if and only if $P$ is obtained by replication of elements of $Q$. Thus, Baclawski's result can 
be extended to this special case of Corollary 6.4

Let $P$ be obtained by replicating elements of $Q$. Then $P$ is $C M$ over $\mathbf{k}$ (SCM over $\mathbf{k}, H C M$ or $S H C M)$ if and only if $Q$ is.

For the case that $P$ and $Q$ are semipure this also follows from Theorems 5.1 and 5.2 .

\section{Subspace arRangements}

The tools from the theory of diagrams of spaces discussed in Section 2 were used by Ziegler and Živaljević [32] to prove results about the homotopy type of various spaces connected to subspace arrangements. In particular, they proved a result (see Theorem 7.3 below) which can be considered a homotopy version and strengthening of the Goresky-MacPherson formula on subspace arrangements. See [3] for a survey of the theory of subspace arrangements.

Since the tools used in [32] are the same as those used to prove Theorem 1.1 it is natural to ask whether one result can be obtained from the other. In this section we show that the Ziegler-Živaljević formula and a related result of Herzog, Reiner and Welker [12] can be viewed as consequences of Theorem 1.1. This does not, however, amount to new proofs, since the methods used are essentially the same.

Let $\Gamma$ be a regular cell complex and let $\Gamma_{1}, \ldots, \Gamma_{n}$ be a collection of subcomplexes whose union is $\Gamma$. For each nonempty subset $I=\left\{i_{1}, \ldots, i_{t}\right\} \subseteq\{1,2, \ldots, n\}$, let $\Gamma_{I}=\Gamma_{i_{1}} \cap \cdots \cap \Gamma_{i_{t}}$. The semilattice of intersections is defined as

$$
L\left(\Gamma_{1}, \ldots, \Gamma_{n}\right):=\left\{\left\|\Gamma_{I}\right\| \mid \emptyset \neq I \subseteq\{1, \ldots, n\}\right\},
$$

ordered by inclusion.

Lemma 7.1 ([12, Lemma 6.1]). Assume that for all nonempty $I, J \subseteq\{1, \ldots, n\}$ the proper inclusion $\Gamma_{I} \subsetneq \Gamma_{J}$ implies $\operatorname{dim} \Gamma_{I}<\operatorname{dim} \Gamma_{J}$, and that each $\Gamma_{I}$ is $\left(\operatorname{dim} \Gamma_{I}-1\right)$ connected. Let $L=L\left(\Gamma_{1}, \ldots, \Gamma_{n}\right) \backslash\{\emptyset\}$. Then

$$
\Gamma \simeq \Delta(L) \vee\left\{T * \Delta\left(L_{>T}\right) \mid T \in L\right\},
$$

where the wedge is formed by identifying each vertex $T$ in the simplicial complex $\Delta(L)$ with a point in the topological space $T * \Delta\left(L_{>T}\right)$.

Proof. The face poset $\mathcal{F}(\Gamma)$ of a regular cell complex $\Gamma$ is the set of closed cells ordered by inclusion. Let $f: \mathcal{F}(\Gamma) \rightarrow L$ send a closed cell $\sigma$ to $\left\|\Gamma_{I}\right\|$, where $\Gamma_{I}$ is the intersection of all $\Gamma_{i}$ containing $\sigma$. Clearly $f$ is order-preserving. We claim that $f$ is well-connected.

Observe that

$$
f^{-1}\left(L_{\leq\left\|\Gamma_{I}\right\|}\right)=\mathcal{F}\left(\Gamma_{I}\right) .
$$

Since a regular cell complex is homeomorphic to the order complex of its face poset 6, Proposition 4.7.8], we have that $\Delta\left(f^{-1}\left(L_{\leq\left\|\Gamma_{I}\right\|}\right)\right)$ is homeomorphic to $\left\|\Gamma_{I}\right\|$, which (by assumption) is $\left(\operatorname{dim} \Gamma_{I}-1\right)$-connected. Since

$$
f^{-1}\left(L_{<\left\|\Gamma_{I}\right\|}\right)=\bigcup_{\Gamma_{J} \subsetneq \Gamma_{I}} \mathcal{F}\left(\Gamma_{J}\right)
$$

and $\ell\left(\mathcal{F}\left(\Gamma_{J}\right)\right)=\operatorname{dim} \Gamma_{J}$, we also have that $\ell\left(f^{-1}\left(L_{<\left\|\Gamma_{I}\right\|}\right)\right)<\operatorname{dim} \Gamma_{I}$. It follows that $\Delta\left(f^{-1}\left(L_{\leq\left\|\Gamma_{I}\right\|}\right)\right)$ is $\ell\left(f^{-1}\left(L_{<\left\|\Gamma_{I}\right\|}\right)\right)$-connected. Hence $f$ is indeed well-connected, and the result follows from Theorem 1.1 . 
Remark 7.2. By using the stronger Theorem [2.5 rather than Theorem 1.1 the conditions in the hypothesis of Lemma 7.1 can be weakened. This results in a stronger form of Lemma [7.1 which is stated in [12, Lemma 6.1].

Let $\mathcal{A}$ be a linear subspace arrangement, i.e., a finite collection of linear subspaces in Euclidean space $\mathbb{R}^{d}$. The singularity link $V_{\mathcal{A}}^{o}$ is defined as

$$
V_{\mathcal{A}}^{o}=S^{d-1} \cap \bigcup_{X \in \mathcal{A}} X
$$

where $S^{d-1}$ is the unit $(d-1)$-sphere in $\mathbb{R}^{d}$. The intersection lattice $L_{\mathcal{A}}$ of $\mathcal{A}$ is the collection of all intersections of subspaces in $\mathcal{A}$ ordered by reverse inclusion. Let $\bar{L}_{\mathcal{A}}$ denote $L_{\mathcal{A}} \backslash\{\hat{0}, \hat{1}\}$ if $\mathcal{A}$ is essential (i.e., $\bigcap \mathcal{A}=\{0\}$ ), and $L_{\mathcal{A}} \backslash\{\hat{0}\}$ otherwise.

Theorem 7.3 (Ziegler \& Živaljević [32]). Let $\mathcal{A}$ be a linear subspace arrangement. Then

$$
V_{\mathcal{A}}^{o} \simeq \Delta\left(\bar{L}_{\mathcal{A}}\right) \vee\left\{\operatorname{susp}^{\operatorname{dim} x}(\Delta(\hat{0}, x)) \mid x \in \bar{L}_{\mathcal{A}}\right\},
$$

where the wedge is formed by identifying each vertex $x$ in $\Delta\left(\bar{L}_{\mathcal{A}}\right)$ with any point in $\operatorname{susp}^{\operatorname{dim} x}(\Delta(\hat{0}, x))$.

Proof. Suppose $\mathcal{A}=\left\{X_{1}, \ldots, X_{n}\right\}$. Let $\mathcal{H}$ be an essential hyperplane arrangement in $\mathbb{R}^{d}$ such that each $X_{i}$ is the intersection of a subcollection of hyperplanes in $\mathcal{H}$. The hyperplane arrangement $\mathcal{H}$ determines a regular cell decomposition of the singularity link $S^{d-1} \cap \bigcup_{X \in \mathcal{H}} X$ (see, e.g., [6, Section 2.1]). Let $\Gamma$ be the subcomplex whose geometric realization is $V_{\mathcal{A}}^{o}$, and for each $i$, let $\Gamma_{i}$ be the subcomplex of $\Gamma$ whose geometric realization is $S^{d-1} \cap X_{i}$. Since the intersection of any $r$-dimensional linear subspace of $\mathbb{R}^{d}$ with $S^{d-1}$ is an $(r-1)$-sphere, $\left\|\Gamma_{I}\right\|$ is a dim $\Gamma_{I^{-}}$-sphere and is therefore $\left(\operatorname{dim} \Gamma_{I}-1\right)$-connected, for each $I$. Since $L_{\mathcal{A}} \backslash\{\hat{0}\}$ is isomorphic to the dual of $L\left(\Gamma_{1}, \ldots, \Gamma_{n}\right)$, the result is obtained by applying Lemma 7.1.

\section{Group aCtions ON HOMOTOPY}

In this section we derive group equivariant versions of Theorem 1.1 and its corollaries. We begin with a review of some definitions.

Let $G$ be a group. A $G$-poset is a poset on which $G$ acts as a group of poset automorphisms. A $G$-poset map $f: P \rightarrow Q$ is a poset map from a $G$-poset $P$ to a $G$-poset $Q$ which commutes with the $G$-action (i.e., $f(g x)=g f(x)$ for all $g \in G$ and $x \in P)$. A $G$-simplicial complex is a simplicial complex on which $G$ acts as a group of simplicial automorphisms. A G-simplicial map $f: \Delta \rightarrow \Gamma$ is a simplicial map from a $G$-simplicial complex $\Delta$ to a $G$-simplicial complex $\Gamma$ which commutes with the $G$-action. A $G$-space is a topological space on which $G$ acts as a group of homeomorphisms. A $G$-continuous map $f: X \rightarrow Y$ from a $G$-space $X$ to a $G$-space $Y$ is a continuous map that commutes with the $G$-action.

Clearly, the order complex of a $G$-poset is a $G$-simplicial complex, and a $G$ poset map induces a $G$-simplicial map. Also, if $\Delta$ is a $G$-simplicial complex, then the induced action of $G$ on the geometric realization $\|\Delta\|$ is a $G$-space and a $G$ simplicial map induces a $G$-continuous map.

Let $f, f^{\prime}: X \rightarrow Y$ be $G$-continuous maps. We say that $f$ and $f^{\prime}$ are $G$-homotopic if there is a homotopy $F: X \times[0,1] \rightarrow Y$ between $f$ and $f^{\prime}$ such that $g F(x, t)=$ $F(g x, t)$ for all $g \in G, x \in X$ and $t \in[0,1]$. Two $G$-spaces $X$ and $Y$ are said to be $G$-homotopy equivalent if there are $G$-continuous maps $\alpha: X \rightarrow Y$ and $\beta: Y \rightarrow X$ 
such that $\alpha \circ \beta$ and $\beta \circ \alpha$ are $G$-homotopic to the respective identity maps on $Y$ and $X$. We denote $G$-homotopy equivalence by $X \simeq_{G} Y$.

A $G$-space $X$ is said to be $G$-contractible if $X$ is $G$-homotopy equivalent to a point. Given a $G$-poset ( $G$-space) $X$, let $X^{G}$ denote the subposet (subspace) of elements (points) fixed by $G$. For $r \geq-1$, a $G$-space $X$ is said to be $(G, r)$-connected if $X^{G}$ is nonempty and for each $G$-simplicial complex $\Delta$ such that $\operatorname{dim} \Delta \leq r$, all $G$-continuous maps from $\|\Delta\|$ to $X$ are $G$-homotopic. Clearly a $G$-contractible space is $(G, r)$-connected for all $r$. An example of an $r$-connected space that is not $(G, r)$-connected is as follows. Let $X$ be a 1 -sphere and let $G$ be the cyclic group generated by the reflection about the line spanned by a pair of antipodal points $a$ and $b$. Although $X$ is 0 -connected, it is not $(G, 0)$-connected. Indeed, the inclusion map from the 0 -sphere consisting of $a$ and $b$ is not $G$-homotopic to the constant map which takes $a$ and $b$ to $a$.

Now let $f: P \rightarrow Q$ be a $G$-poset map. Assume that $f^{-1}\left(Q_{\leq q}\right)^{\operatorname{Stab}_{G}(q)}$ is nonempty for all $q \in Q$, and choose $c_{q} \in f^{-1}\left(Q_{\leq q}\right)^{\operatorname{Stab}_{G}(q)}$ so that $g c_{q}=c_{g q}$ for all $g \in G$. This can be done by first choosing the $c_{q}$ 's for the orbit representatives in $Q$. With a fixed choice of $c_{q}$ 's we can form the $G$-simplicial complex

$$
\Gamma\left(f,\left\{c_{q}\right\}_{q \in Q}\right):=\Delta(Q) \vee\left\{\Delta\left(f^{-1}\left(Q_{\leq q}\right)\right) * \Delta\left(Q_{>q}\right) \mid q \in Q\right\},
$$

where the wedge is formed by identifying each $q \in Q$ with $c_{q} \in f^{-1}\left(Q_{\leq q}\right)$. The action of $G$ on the vertex set $\biguplus_{q \in Q}\left(f^{-1}\left(Q_{\leq q}\right) \uplus Q_{>q}\right)$ of $\Gamma\left(f,\left\{c_{q}\right\}_{q \in Q}\right)$ can be described as follows: If $x \in f^{-1}\left(Q_{\leq q}\right) \uplus Q_{>q}$ then $g \in G$ takes $x$ to $g x$ in $f^{-1}\left(Q_{\leq g q}\right) \uplus$ $Q_{>g q}$.

Theorem 8.1. Let $f: P \rightarrow Q$ be a G-poset map such that for all $q \in Q$ the fiber $f^{-1}\left(Q_{\leq q}\right)$ is $\left(\operatorname{Stab}_{G}(q), \ell\left(f^{-1}\left(Q_{<q}\right)\right)\right)$-connected. Then

$$
\Delta(P) \simeq_{G} \Delta(Q) \vee\left\{\Delta\left(f^{-1}\left(Q_{\leq q}\right)\right) * \Delta\left(Q_{>q}\right) \mid q \in Q\right\},
$$

where the wedge is formed by identifying each $q \in Q$ with $c_{q} \in f^{-1}\left(Q_{\leq q}\right)$, where the $c_{q}$ are chosen so that $g c_{q}=c_{g q}$.

The proof of Theorem 8.1 goes along the lines of the proof of Theorem 1.1 using an equivariant version of a $Q$-diagram (see Definition 9.7) and equivariant versions of the Projection Lemma, Homotopy Lemma, Wedge Lemma (see for example [29]) and the Homotopy Extension Property.

Theorem 8.1 generalizes the equivariant Quillen fiber lemma, which was first proved and applied by Thévenaz and Webb 23].

Corollary 8.2 ([23]). Let $f: P \rightarrow Q$ be a G-poset map such that for all $q \in Q$ the fiber $\Delta\left(f^{-1}\left(Q_{\leq q}\right)\right)$ is $\operatorname{Stab}_{G}(q)$-contractible. Then $\Delta(P)$ and $\Delta(Q)$ are $G$-homotopy equivalent.

Equivariant versions of all the corollaries in Section 3 follow from Theorem 8.1 We state the equivariant version of Corollary 3.5.

Corollary 8.3. Let $f: \Gamma \rightarrow \Delta$ be a G-simplicial map. If the fiber $f^{-1}(\dot{F})$ is $\left(\operatorname{Stab}_{G}(F), \operatorname{dim} f^{-1}(\dot{F} \backslash\{F\})\right)$-connected for all nonempty faces $F$ of $\Delta$, then

$$
\Gamma \simeq_{G} \Delta \vee\left\{f^{-1}(\dot{F}) * \mathrm{lk}_{\Delta} F \mid F \in \Delta \backslash\{\emptyset\}\right\},
$$

where the wedge is formed by identifying a vertex $c_{F}$ in $f^{-1}(\dot{F})$ with a vertex of $F$ and the $c_{F}$ are chosen so that $g c_{F}=c_{g F}$ for all $g \in G$. 
It is clear from the proof of Theorem 8.1 that the equivariant connectivity assumption can be replaced by the weaker assumption that the inclusion map $\Delta\left(f^{-1}\left(Q_{<q}\right)\right) \hookrightarrow \Delta\left(f^{-1}\left(Q_{\leq q}\right)\right)$ is $\operatorname{Stab}_{G}(q)$-homotopic to the constant map. Even this assumption seems to be very strong, and we do not see an application of the result in its full strength. The following example shows that an equivariant connectivity assumption is needed.

Example 8.4. Let $f: P \rightarrow Q$ be the well-connected poset map discussed in Example 1.2. Let $G$ be the cyclic group of order 2 whose non-identity element acts by $(12)(34)$ on $P$ and trivially on $Q$. Note that if $q$ is one of the maximal elements of $Q$, then the fiber $f^{-1}\left(Q_{\leq q}\right)$ is $G$-homeomorphic to a circle with (1 2)(3 4) acting by reflecting the circle about the line spanned by a pair of antipodal points. As was previously observed, this $G$-space is not $(0, G)$-connected. We now see that (8.1) does not hold. Clearly, $\Delta(P)$ is $G$-homeomorphic to two circles intersecting in two points such that these two points are the only fixed points and $\left(\begin{array}{ll}1 & 2\end{array}\right)\left(\begin{array}{ll}3 & 4\end{array}\right)$ reflects each circle about the line spanned by the fixed points. The $G$-complex on the right side of (8.1) has a circle attached to each element of $Q$. One of the circles is fixed by (12)(34), and each of the other two circles is reflected about the line spanned by the wedge point and its antipode. Although the simplicial complexes are homotopy equivalent, they fail to be $G$-homotopy equivalent. To be $G$-homotopy equivalent the subcomplexes of points that are fixed by the action of $G$ must be homotopy equivalent. The fixed point subcomplex of $\Delta(P)$ consists of two isolated points, and the fixed point subcomplex of the right side of (8.1) has the homotopy type of the wedge of a 1 -sphere and two 0 -spheres.

\section{Group aCtions on HOMOLOGY}

Although the strong assumptions dilute the applicability of Theorem 8.1, it is possible to prove a result for the $G$-module structure of the homology groups without such restrictions. The action of $G$ on a simplicial complex $\Delta$ induces a representation of $G$ on reduced simplicial homology $\tilde{H}_{*}(\Delta ; \mathbf{k})$, where $\mathbf{k}$ is any field. For the remainder of this paper we assume that $\mathbf{k}$ is a field of characteristic 0 .

Given a subgroup $H$ of $G$ and a $\mathbf{k} H$-module $V$, let $V \uparrow_{H}^{G}$ denote the induction of $V$ to $G$.

Theorem 9.1. Fix a nonnegative integer $t$. Let $f: P \rightarrow Q$ be a $G$-poset map such that for all $q \in Q$ the fiber $\Delta\left(f^{-1}\left(Q_{\leq q}\right)\right)$ is $\min \left\{t, \ell\left(f^{-1}\left(Q_{<q}\right)\right)\right\}$-acyclic over the field $\mathbf{k}$. Then for all $r \leq t$, we have the following isomorphism of $\mathbf{k} G$-modules:

$$
\begin{aligned}
& \tilde{H}_{r}(P ; \mathbf{k}) \\
& \quad \cong_{G} \tilde{H}_{r}(Q ; \mathbf{k}) \oplus \bigoplus_{q \in Q / G} \bigoplus_{i=-1}^{r}\left(\tilde{H}_{i}\left(f^{-1}\left(Q_{\leq q}\right) ; \mathbf{k}\right) \otimes \tilde{H}_{r-i-1}\left(Q_{>q} ; \mathbf{k}\right)\right) \uparrow_{\operatorname{Stab}_{G}(q)}^{G} .
\end{aligned}
$$

Before proving the theorem, we consider an example and some consequences.

Example 9.2. Theorem 9.1 can be applied to the poset map given in Example 8.4 View $G$ as the symmetric group $\mathfrak{S}_{2}$. The conclusion is that $\tilde{H}_{r}(P ; \mathbf{k})$ is 0 unless $r=1$, in which case the $\mathfrak{S}_{2}$-module $\tilde{H}_{1}(P ; \mathbf{k})$ decomposes into $S^{2} \oplus S^{1^{2}} \oplus S^{1^{2}}$, where $S^{\lambda}$ denotes the irreducible representation of $\mathfrak{S}_{n}$ indexed by $\lambda$. The first summand comes from the bottom element of $Q$ and the other two summands come from the top elements. 
The following "equivariant homology Quillen fiber lemma" is a direct consequence of the theorem.

Corollary 9.3. Let $f: P \rightarrow Q$ be a G-poset map. If the fiber $\Delta\left(f^{-1}\left(Q_{\leq q}\right)\right)$ is $t$-acyclic over $\mathbf{k}$ for all $q \in Q$, then, as $G$-modules,

$$
\tilde{H}_{r}(P ; \mathbf{k}) \cong_{G} \tilde{H}_{r}(Q ; \mathbf{k}),
$$

for all $r \leq t$.

Equivariant homology versions of all the consequences of Theorem 1.1 discussed in previous sections follow from Theorem 9.1 We state two of these equivariant homology results here.

Corollary 9.4. Let $f: \Gamma \rightarrow \Delta$ be a G-simplicial map. If the fiber $f^{-1}(\dot{F})$ is $\min \left\{t, \operatorname{dim} f^{-1}(\dot{F} \backslash\{F\})\right\}$-acyclic over $\mathbf{k}$ for all nonempty faces $F$ of $\Delta$, then for all $r \leq t$,

$$
\tilde{H}_{r}(\Gamma ; \mathbf{k}) \cong_{G} \bigoplus_{F \in \Delta / G} \bigoplus_{i=-1}^{r}\left(\tilde{H}_{i}\left(f^{-1}(\dot{F}) ; \mathbf{k}\right) \otimes \tilde{H}_{r-i-1}\left(\mathrm{lk}_{\Delta} F ; \mathbf{k}\right)\right) \uparrow_{\operatorname{Stab}_{G}(F)}^{G} .
$$

Corollary 9.5. Let $\Delta$ be a G-simplicial complex on vertex set $[n]$ and let $\mathbf{m}$ be an $n$-sequence of positive integers. If $G$ acts on the inflation $\Delta_{\mathbf{m}}$ and this action commutes with the deflating map, then for all $r \in \mathbb{Z}$,

$$
\tilde{H}_{r}\left(\Delta_{\mathbf{m}} ; \mathbf{k}\right) \cong_{G} \bigoplus_{F \in \Delta / G}\left(\tilde{H}_{|F|-1}\left(\dot{F}_{\mathbf{m}(F)} ; \mathbf{k}\right) \otimes \tilde{H}_{r-|F|}\left(\mathrm{lk}_{\Delta} F ; \mathbf{k}\right)\right) \uparrow_{\operatorname{Stab}_{G}(F)}^{G},
$$

where $\mathbf{m}(F)$ is the subsequence $\left(m_{i_{1}}, \ldots, m_{i_{t}}\right)$ of $\mathbf{m}=\left(m_{1}, \ldots, m_{n}\right)$ for $F=\left\{i_{1}<\right.$ $\left.\cdots<i_{t}\right\}$.

The following is a homology version of a generalization of [4, Lemma 11.12] and [23, Proposition 1.7].

Corollary 9.6. Let $P$ be a $G$-poset and $A$ a $G$-invariant induced subposet of $P$ such that $\Delta\left(P_{<x}\right)$ is $t$-acyclic for all $x \in P \backslash A$. Then

$$
\tilde{H}_{r}(A) \cong_{G} \tilde{H}_{r}(P),
$$

for all $r \leq t$.

Proof. The proof is similar to that of [23, Proposition 1.7]. We use the embedding map $f: P \backslash M \rightarrow P$, where $M$ is the set of maximal elements of $P \backslash A$.

The proof of Theorem 9.1 follows the lines of the proof of Theorem 4.1 using an equivariant version of Corollary [2.4 (cf. [22]) and the equivariant version of Proposition 4.2 given in Proposition 9.8 below.

Definition 9.7. Given a $G$-poset $Q$, a (simplicial) $Q$-diagram $\mathcal{D}$ is said to be a (simplicial) $(G, Q)$-diagram if $\biguplus_{q \in Q} D_{q}$ is a $G$-space (simplicial complex) satisfying

- $g D_{q}=D_{g q}$ for all $g \in G$ and $q \in Q$, and

- $g d_{x, y}(a)=d_{g x, g y}(g a)$ for all $x \leq_{Q} y, a \in D_{x}$ and $g \in G$.

The action of $G$ on $\biguplus_{q \in Q} D_{q}$ induces natural actions of $G$ on colim $\mathcal{D}$ and hocolim $\mathcal{D}$. 
Proposition 9.8 ([22, Proposition 2.3]). Let $\mathcal{D}$ be a simplicial $(G, Q)$-diagram for which each $D_{x} \neq\{\emptyset\}$. Let $t$ be a nonnegative integer. Assume that for all nonminimal $y$ in $Q$ and $r \leq t$, the induced map

$$
\left(\cup_{x<y} d_{x, y}\right)^{*}: \tilde{H}_{r}\left(\biguplus_{x<y} D_{x} ; \mathbf{k}\right) \rightarrow \tilde{H}_{r}\left(D_{y} ; \mathbf{k}\right)
$$

is trivial. Then for all $r \leq t$,

$$
\begin{aligned}
& \tilde{H}_{r}(\text { hocolim } \mathcal{D} ; \mathbf{k}) \\
& \quad \cong_{G} \tilde{H}_{r}(Q ; \mathbf{k}) \oplus \bigoplus_{x \in Q / G} \bigoplus_{i=-1}^{r}\left(\tilde{H}_{i}\left(D_{x} ; \mathbf{k}\right) \otimes \tilde{H}_{r-i-1}\left(Q_{>x} ; \mathbf{k}\right)\right) \uparrow_{\operatorname{Stab}_{G}(x)}^{G} .
\end{aligned}
$$

Proof. Let $\left(C_{r}(\text { hocolim } \mathcal{D} ; \mathbf{k}), \delta_{r}\right)_{r=0, \ldots, d}$, where $d=\operatorname{dim}($ hocolim $\mathcal{D})$, denote the cellular chain complex of the CW-complex hocolim $\mathcal{D}$. The cells of hocolim $\mathcal{D}$ are of the form

$$
\alpha \times(\{x\} * \beta),
$$

where $x \in Q, \alpha \in D_{x} \backslash\{\emptyset\}$ and $\beta \in \Delta\left(Q_{>x}\right)$. Let $\min \beta$ denote the smallest element of the chain $\beta$. The differential is given by

$$
\delta(\alpha \times(\{x\} * \beta))=A+B+C,
$$

where

$$
\begin{gathered}
A= \begin{cases}\partial(\alpha) \times(\{x\} * \beta) & \text { if } \operatorname{dim} \alpha>0, \\
0 & \text { otherwise, }\end{cases} \\
B= \begin{cases}(-1)^{\ell(\alpha)-1} d_{x, \min \beta}(\alpha) \times \beta & \text { if } \operatorname{dim} d_{x, \min \beta}(\alpha)=\operatorname{dim} \alpha, \\
0 & \text { otherwise, }\end{cases} \\
C=(-1)^{\ell(\alpha)} \alpha \times(\{x\} * \partial(\beta)),
\end{gathered}
$$

and $\partial$ is the simplicial boundary map. We use the theory of spectral sequences to compute the homology of the cellular chain complex $\left(C_{r}(\text { hocolim } \mathcal{D} ; \mathbf{k}), \delta_{r}\right)_{r=0, \ldots, d}$. For $r, m=0, \ldots, d$, let $F_{r, m}$ be the subspace of $C_{r}($ hocolim $\mathcal{D} ; \mathbf{k})$ spanned by the $r$-dimensional cells for which the chain $\beta$ has length at most $m-1$. Clearly the $F_{r, m}$ are $G$-invariant and $\delta_{r} F_{r, m} \subseteq F_{r-1, m}$. So

$$
F_{r,-1} \subseteq F_{r, 0} \subseteq \cdots \subseteq F_{r, r}=C_{r}(\text { hocolim } \mathcal{D} ; \mathbf{k})
$$

is a filtration of the complex of $\mathbf{k} G$-modules $\left(C_{r}(\right.$ hocolim $\left.\mathcal{D} ; \mathbf{k}), \delta_{r}\right)$. In the spectral sequence associated with this filtration, the $E^{1}$ component is given by $E_{r, m}^{1}=$ $H_{r}\left(F_{r, m}, F_{r-1, m-1} ; \mathbf{k}\right)$. It is clear that if $\alpha \times(x * \beta) \in F_{r, m}$, then $B$ and $C$ of (9.2) are in $F_{r-1, m-1}$. It follows that $E_{r, m}^{1}$ is generated by elements of the form

$$
\alpha \times(\{x\} * \beta),
$$

where $x \in Q, \alpha \in H_{r-m}\left(D_{x} ; \mathbf{k}\right)$ and $\beta$ is a chain of length $m-1$ in $Q_{>x}$. The differential $\delta^{1}: E_{r, m}^{1} \rightarrow E_{r-1, m-1}^{1}$ is given by

$$
\delta^{1}(\alpha \times(\{x\} * \beta))=B^{*}+C,
$$

where $B^{*}$ is like $B$ in 9.3 except that $d_{x, \min \beta}$ is replaced by the induced map $d_{x, \min \beta}^{*}$ and $C$ is given by 9.4 . 
If $m<r \leq t$ and $\alpha \in H_{r-m}\left(D_{x} ; \mathbf{k}\right)$, then $\alpha$ is also in the reduced homology $\tilde{H}_{r-m}\left(D_{x} ; \mathbf{k}\right)$. Hence $d_{x, \min \beta}^{*}(\alpha)=0$ by (9.1). It follows that $B^{*}=0$, and so

$$
\delta^{1}(\alpha \times(\{x\} * \beta))=(-1)^{\ell(\alpha)} \alpha \times(\{x\} * \partial(\beta)) .
$$

We can see that, as $G$-modules,

$$
E_{r, m}^{1} \cong_{G} \bigoplus_{x \in Q / G}\left(\tilde{H}_{r-m}\left(D_{x}, \mathbf{k}\right) \otimes \tilde{C}_{m-1}\left(Q_{>x} ; \mathbf{k}\right)\right) \uparrow_{\operatorname{Stab}_{G}(x)}^{G},
$$

and that $E_{r, m}^{2}$, the homology of the complex $\left(E_{r, m}^{1}, \delta_{r, m}^{1}\right)$, is isomorphic to the $G$-module

$$
\bigoplus_{x \in Q / G} \tilde{H}_{m-1}\left(Q_{>x} ; \tilde{H}_{r-m}\left(D_{x} ; \mathbf{k}\right)\right) \uparrow_{\operatorname{Stab}_{G}(x)}^{G} .
$$

By the Universal Coefficient Theorem we have the $G$-module isomorphism

$$
E_{r, m}^{2} \cong_{G} \bigoplus_{x \in Q / G}\left(\tilde{H}_{m-1}\left(Q_{>x} ; \mathbf{k}\right) \otimes \tilde{H}_{r-m}\left(D_{x} ; \mathbf{k}\right)\right) \uparrow_{\operatorname{Stab}_{G}(x)}^{G}
$$

Now we compute $E_{r, m}^{2}$ for $r=m$. For each $x$ that is minimal in $Q$, set

$$
m_{x}:=\frac{1}{\left|V\left(D_{x}\right)\right|} \sum_{v \in V\left(D_{x}\right)}[v] \in H_{0}\left(D_{x} ; \mathbf{k}\right),
$$

where $V\left(D_{x}\right)$ denotes the vertex set of the simplicial complex $D_{x}$ and [.] denotes (nonreduced) homology class. Note that we are using the fact that $\mathbf{k}$ has characteristic 0 here. It is clear that $g m_{x}=m_{g x}$ for all minimal $x$ and $g \in G$. Now, let $y$ be a non-minimal element of $Q$. Let

$$
d_{y}:=\bigcup_{x<y} d_{x, y}
$$

It follows from the fact that $d_{y}^{*}$ is trivial on the reduced homology $\tilde{H}_{0}\left(\biguplus_{x<y} D_{x} ; \mathbf{k}\right)$ that if $a$ and $b$ are points in $\biguplus_{x<y} D_{x}$, then

$$
d_{y}^{*}([a])=d_{y}^{*}([b]) .
$$

(Here $d_{y}^{*}$ is the induced map on nonreduced homology, and reduced homology is viewed as a submodule of nonreduced homology.) It follows from (9.7) that $d_{y}^{*}\left(m_{x_{1}}\right)=d_{y}^{*}\left(m_{x_{2}}\right)$ for all minimal elements $x_{1}, x_{2}<y$. This allows us to define $m_{y}$ to be the common value of $d_{y}^{*}\left(m_{x}\right)$ for all minimal $x<y$. Note that this construction also implies

$$
d_{y}^{*}\left(m_{x}\right)=m_{y}
$$

for all $x<y$, not just the minimal $x$. We also need to note that $g m_{y}=m_{g y}$ for all $y \in Q$ and $g \in G$.

For each $x \in Q$ we can decompose $H_{0}\left(D_{x} ; \mathbf{k}\right)$ into the direct sum of the subspace $\tilde{H}_{0}\left(D_{x} ; \mathbf{k}\right)$ and the subspace generated by $m_{x}$. This enables us to decompose $E_{r, r}^{1}$ into $G$-invariant subspaces $U_{r}$ and $V_{r}$. The subspace $U_{r}$ is generated by elements of the form $\alpha \times(\{x\} * \beta)$, where $x \in Q, \alpha \in \tilde{H}_{0}\left(D_{x} ; \mathbf{k}\right)$ and $\beta$ is a chain of length $r-1$ in $Q_{>x}$. The subspace $V_{r}$ is generated by elements of the form $m_{x} \times(\{x\} * \beta)$, 
where $x \in Q$ and $\beta$ is a chain of length $r-1$ in $Q_{>x}$. Let $H_{r}(U)$ be the homology of the complex $\left(U_{r}, \delta_{r}^{1}\right)$. Just as for the case $r>m$, we have

$$
H_{r}(U) \cong_{G} \bigoplus_{x \in Q / G}\left(\tilde{H}_{r-1}\left(Q_{>x} ; \mathbf{k}\right) \otimes \tilde{H}_{0}\left(D_{x} ; \mathbf{k}\right)\right) \uparrow_{\operatorname{Stab}_{G}(x)}^{G} .
$$

Let $\phi_{r}: V_{r} \rightarrow C_{r}(Q ; \mathbf{k})$ be the $G$-isomorphism defined by

$$
\phi_{r}\left(m_{x} \times(\{x\} * \beta)\right)=\{x\} * \beta .
$$

It follows from the fact that $d_{\min \beta}^{*}\left(m_{x}\right)=m_{\min \beta}$ (cf. (9.8)) that $\phi_{r}$ commutes with the differentials $\delta_{r}^{1}$ and $\partial_{r}$. Hence, the homology $H_{r}(V)$ of the complex $\left(V_{r}, \delta_{r}^{1}\right)$ is given by

We now have

$$
H_{r}(V) \cong_{G} \tilde{H}_{r}(Q ; \mathbf{k}) .
$$

$$
E_{r, r}^{2} \cong_{G} \tilde{H}_{r}(Q ; \mathbf{k}) \oplus \bigoplus_{x \in Q / G}\left(\tilde{H}_{r-1}\left(Q_{>x} ; \mathbf{k}\right) \otimes \tilde{H}_{0}\left(D_{x} ; \mathbf{k}\right)\right) \uparrow_{\operatorname{Stab}_{G}(x)}^{G}
$$

It is easily seen that $\delta^{2}=0$, and thus the result follows.

Remark 9.9. Proposition 9.8 is a slight generalization of Proposition 2.3 of Sundaram and Welker [22]. The proof given above is essentially that of Sundaram and Welker [22], with some details filled in. We include this proof in order to account for the term $\tilde{H}_{r}(Q ; \mathbf{k})$, which is missing from their decomposition (a correct statement is given in [29, Theorem 8.11]). Note that if there is no group action involved, then it is not necessary to assume that $\mathbf{k}$ has characteristic 0 , because one can simply define $m_{x}$, for minimal $x$, to be the homology class of any point in $D_{x}$.

Sundaram and Welker [22] use Proposition 9.8 to derive an equivariant homology version of the Ziegler-Živaljević formula (7.1). The Sundaram-Welker formula can also be viewed as a consequence of Theorem 9.1 just as the Ziegler-Živaljević formula was viewed as a consequence of Theorem 1.1 in Section 7

\section{REFERENCES}

[1] E. K. Babson, A combinatorial flag space, Ph. D. Thesis, MIT, 1993.

[2] K. Baclawski, Cohen-Macaulay ordered sets, J. Algebra 63 (1980), 226-258. MR 81m:06002

[3] A. Björner, Subspace arrangements, First European Congress of Mathematics, Paris 1992, A. Joseph et al. (Eds), Progress in Math., 119, Birkhäuser, 1994, pp. 321-370. MR 96h:52012

[4] A. Björner, Topological Methods, Handbook of Combinatorics, R. Graham, M. Grötschel and L. Lovász, (Eds), North-Holland, Amsterdam, 1995, pp. 1819-1872. MR 96m:52012

[5] A. Björner, Nerves, fibers and homotopy groups, J. Combin. Theory, Ser. A, 102 (2003), 88-93. MR 2004a:55018

[6] A. Björner, M. Las Vergnas, B. Sturmfels, N. White, and G.M. Ziegler, Oriented Matroids, Encyclopedia of Mathematics and its Applications, Cambridge University Press, Cambridge, 1993. Second edition, 1999. MR 95e:52023. MR 2000j:52016

[7] A. Björner and M.L. Wachs, Shellable nonpure complexes and posets, I, Trans. AMS 348 (1996), 1299-1327. MR 96i:06008

[8] A. Björner, M.L. Wachs and V. Welker, On sequentially Cohen-Macaulay complexes and posets, in preparation.

[9] A. Björner and V. Welker, Segre and Rees products of posets, with ring-theoretic applications, preprint, 2003 (http://arxiv.org/abs/math.CO/0312516).

[10] G.E. Bredon, Topology and Geometry, Graduate Texts in Mathematics, 139, Springer-Verlag, New York-Heidelberg-Berlin, 1993. MR 94d:55001

[11] A. Hatcher, Algebraic Topology, Cambridge University Press, 2001. MR 2002k:55001

[12] J. Herzog, V. Reiner and V. Welker, The Koszul property in affine semigroup rings, Pacific J. Math. 186 (1998), 39-65. MR 99i:13010 
[13] P.J. Hilton, An Introduction to Homotopy Theory, Cambridge Tracts in Mathematics and Mathematical Physics, 43, Cambridge University Press, 1953. MR 15:52c

[14] B. Mirzaii and W. van der Kallen, Homology stability for unitary groups, Documenta Math. 7 (2002), 143-166. MR 2003e:19007

[15] J. Pakianathan and E. Yalçin, On commuting and non-commuting complexes, J. Algebra 236 (2001), 396-418. MR 2002c:20036

[16] J.S. Provan and L.J. Billera, Decompositions of simplicial complexes related to diameters of convex polyhedra, Math. Oper. Res. 5 (1980), 576-594. MR 82c:52010

[17] D. Quillen, Homotopy properties of the poset of non-trivial p-subgroups of a group, Advances in Math. 28 (1978), 101-128 MR 80k:20049

[18] J. Shareshian, Some results on hypergraph matching complexes and p-group complexes of symmetric groups, preprint, 2000.

[19] J. Shareshian and M.L. Wachs, On the top homology of hypergraph matching complexes, in preparation.

[20] R.P. Stanley, Combinatorics and Commutative Algebra, Second edition, Birkhäuser, Boston, 1995. MR 98h:05001

[21] B. Sturmfels and G.M. Ziegler, Extension spaces of oriented matroids, Discrete Comput. Geometry 10 (1993), 23-45. MR 94i:52015

[22] S. Sundaram and V. Welker, Group actions on arrangements and applications to configuration spaces, Trans. Amer. Math. Soc. 349 (1997), 1389-1420. MR 97h:52012

[23] J. Thévenaz and P.J. Webb, Homotopy equivalence of posets with a group action J. Combin. Theory, Ser. A 56 (1991), 173-181. MR 92k:20049

[24] M.L. Wachs, Whitney homology of semipure shellable posets, J. Algebraic Combinatorics 9 (1999), 173-207. MR 2000e:06004

[25] M.L. Wachs, Topology of matching, chessboard, and general bounded degree graph complexes, Algebra Universalis, Special Issue in Memory of Gian-Carlo Rota, 49 (2003), 345-385.

[26] M.L. Wachs, Bounded degree digraph and multigraph matching complexes, in preparation.

[27] M.L. Wachs, Poset fiber theorems and Dowling lattices, in preparation.

[28] J.W. Walker, Homotopy type and Euler characteristic of partially ordered sets, Europ. J. Combinatorics 2 (1981), 373-384. MR 83g:06002

[29] V. Welker, Partition Lattices, Group Actions on Arrangements and Combinatorics of Discriminants, Habilitationsschrift, Essen, 1996.

[30] V. Welker, G.M. Ziegler and R.T. Živaljević, Homotopy colimits - comparison lemmas for combinatorial applications, J. Reine Angew. Mathematik (Crelles Journal) 509 (1999), 117 149. MR 2000b:55010

[31] P.J. Witbooi, Excisive triads and double mapping cylinders, Topology and its Applications 95(1999), 169-172. MR 2000d:55026

[32] G.M. Ziegler and R.T. Živaljević, Homotopy type of arrangements via diagrams of spaces, Math. Ann. 295 (1983), 527-548. MR 94c:55018

Department of Mathematics, Royal Institute of Technology, S-100 44 Stockholm, SWEDEN

E-mail address: bjorner@math.kth.se

Department of Mathematics, University of Miami, Coral Gables, Florida 33124

E-mail address: wachs@math.miami.edu

Fachbereich Mathematik und Informatik, Universität Marburg, D-350 32 Marburg, GERMANY

E-mail address: welker@mathematik.uni-marburg.de 